\title{
THE IMPACT OF AGRICULTURAL POLICIES ON PRODUCING THE MOST IMPORTANT STRATEGICAL CROPS IN EGYPT
}

Aly, A. I.

Department of Agricultural Economics, Faculty of Agric., El - Fayoum

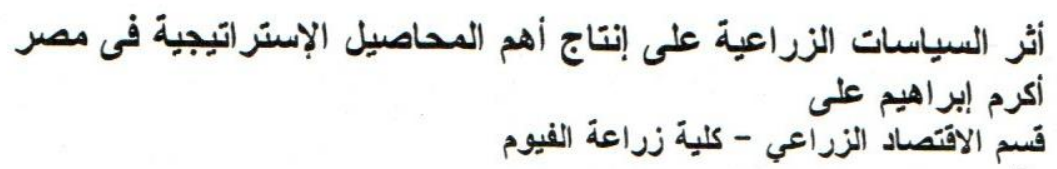

الملخـصـ

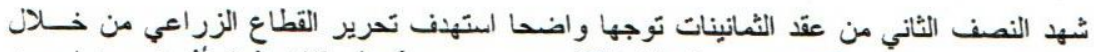

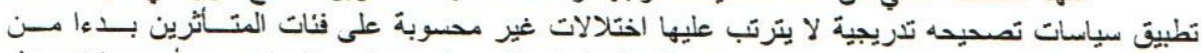

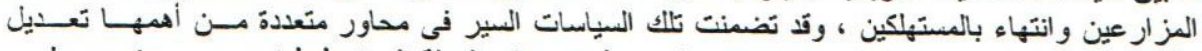

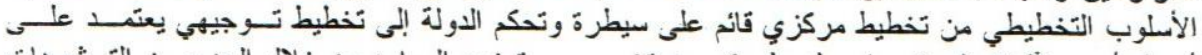

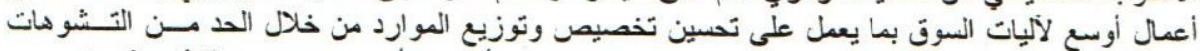

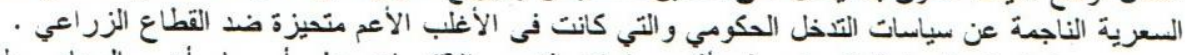

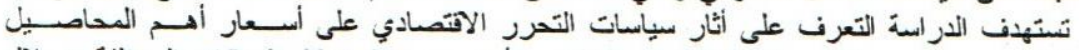

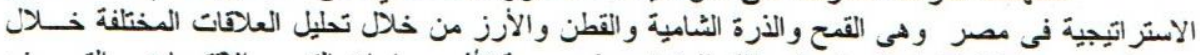

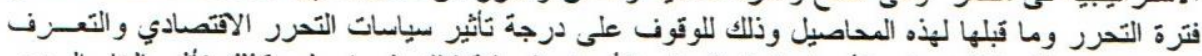

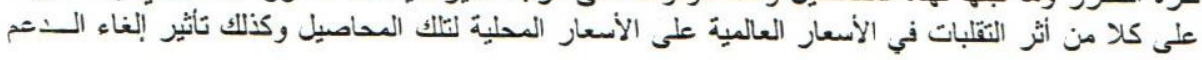

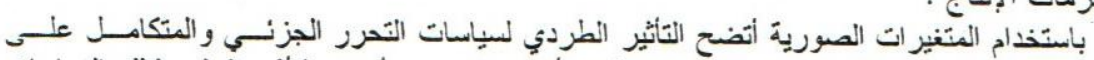

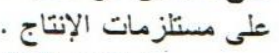

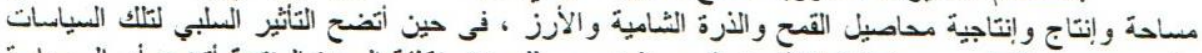

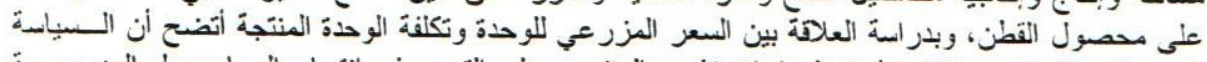

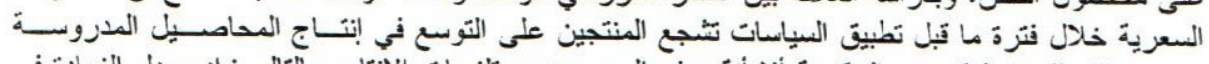

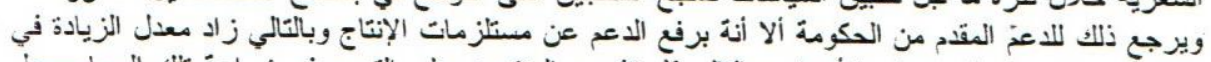

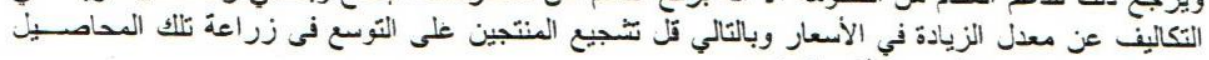

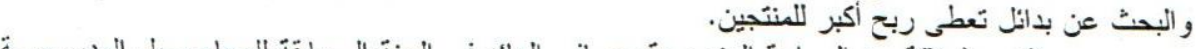

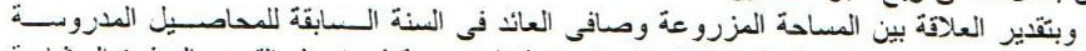

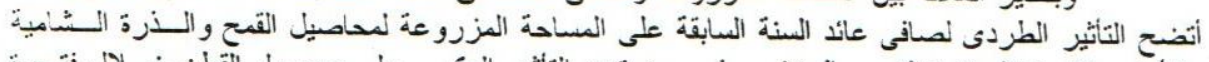

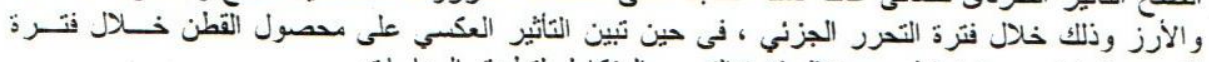

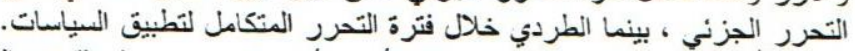

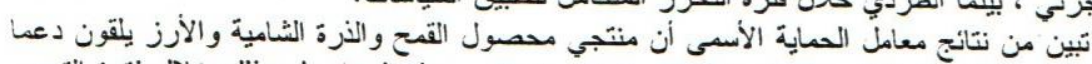

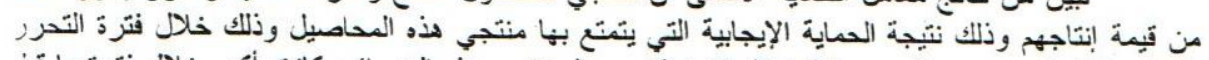

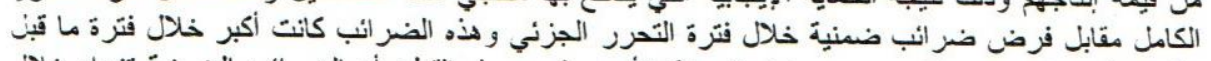

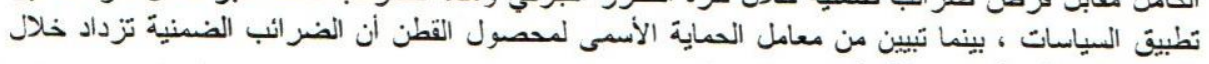

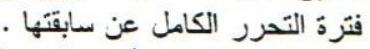

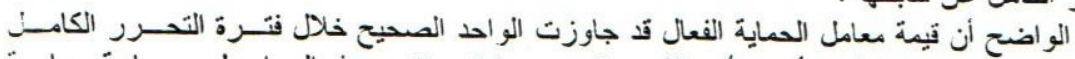

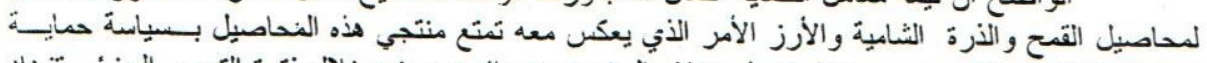

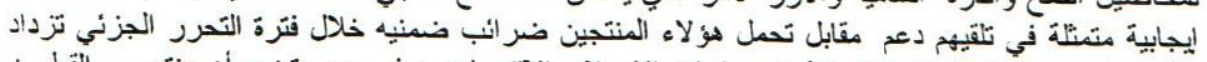

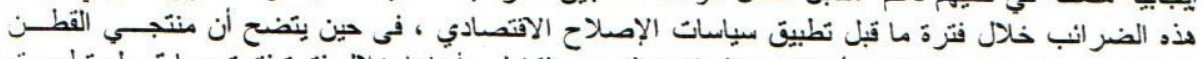

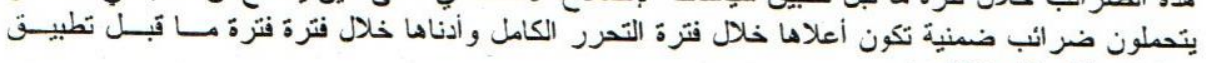




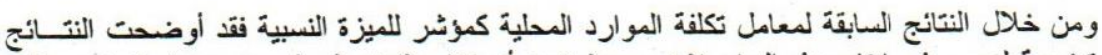

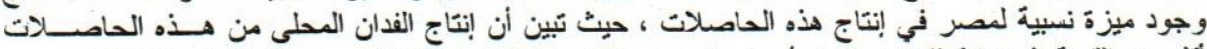

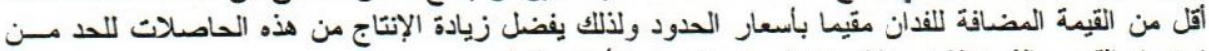

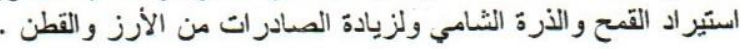

\section{المقدمة}

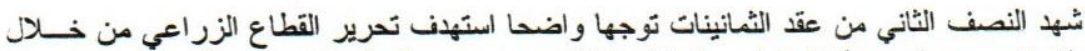

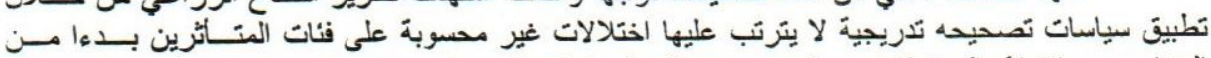

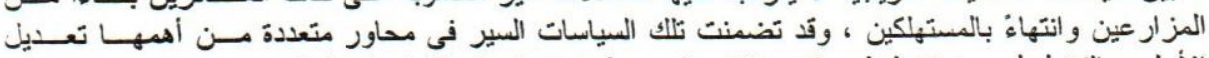

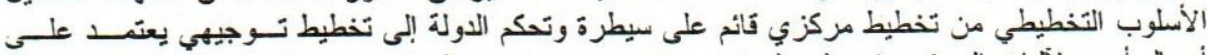

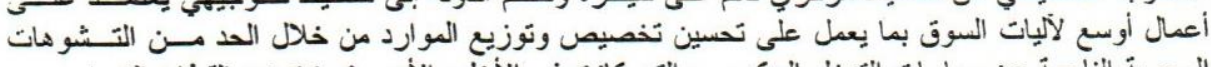

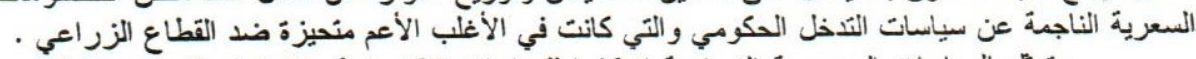

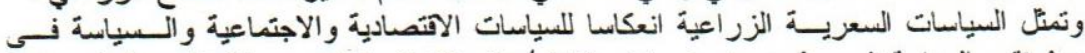

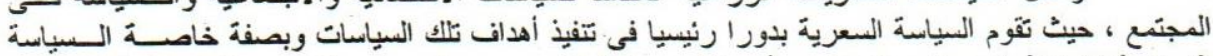

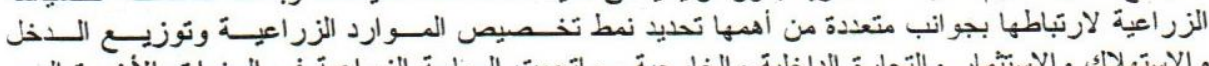

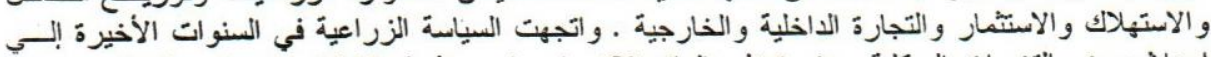

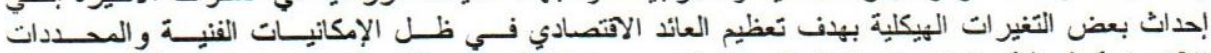

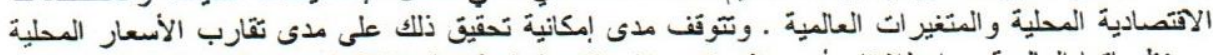

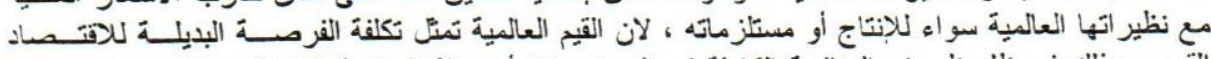

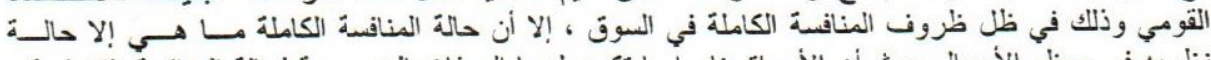

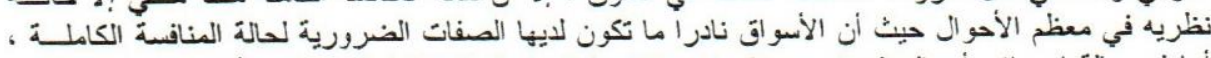

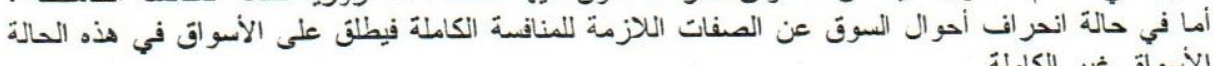

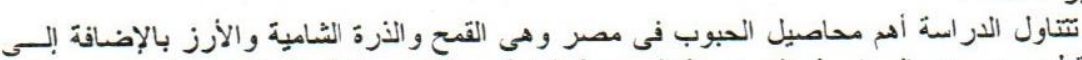

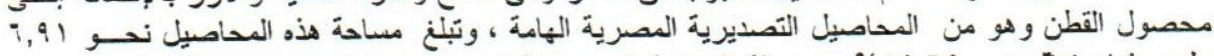

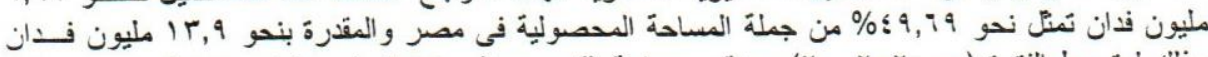

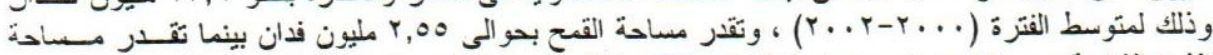

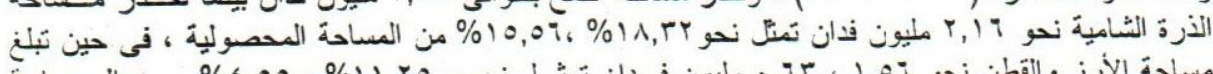

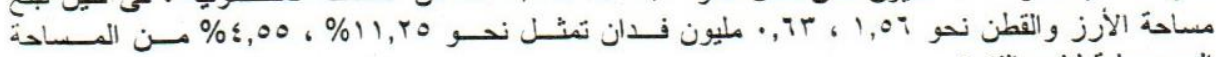
المحصولية لنفس الفترة.

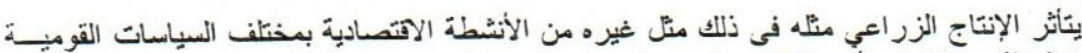

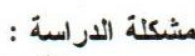

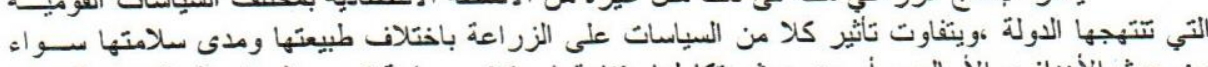

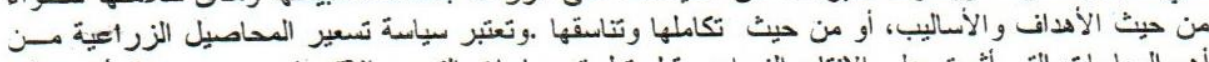

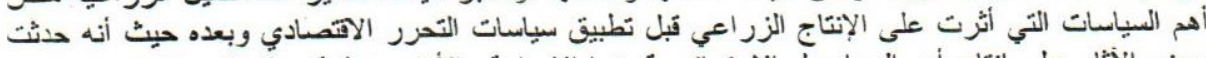

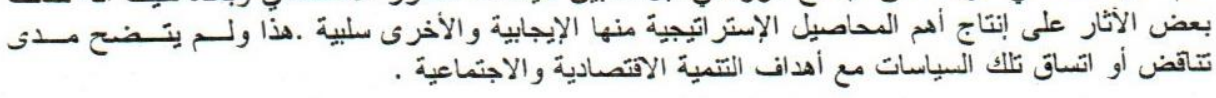

الهوف من الاراسة : التران

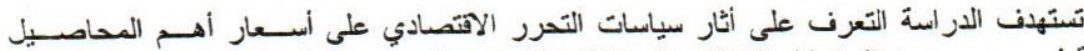

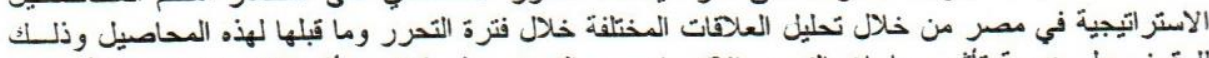

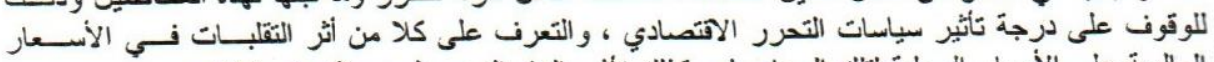

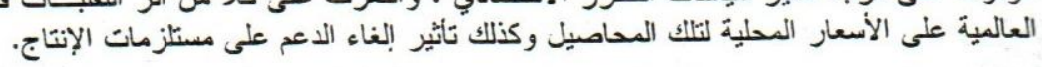




\section{J. Agric. Sci. Mansoura Univ., 30 (7), July, 2005}

\section{الطريقة البحثية ومصادر البيانات}

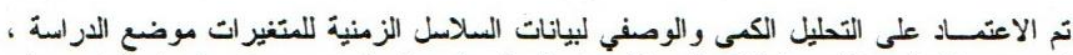

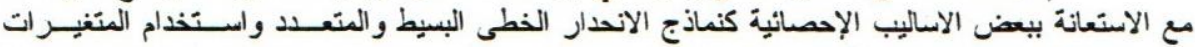

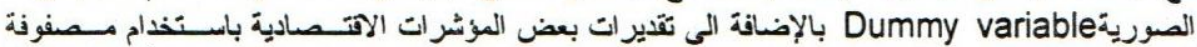

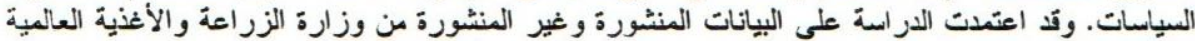

. FAO

\section{مناقشة النتانج}

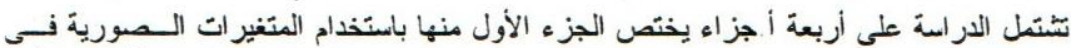

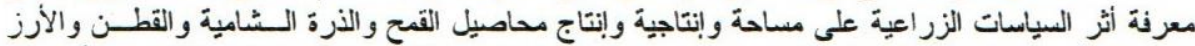

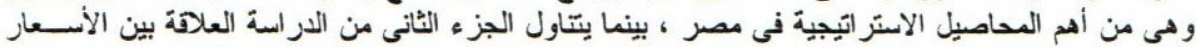

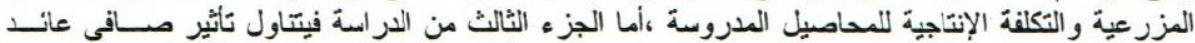

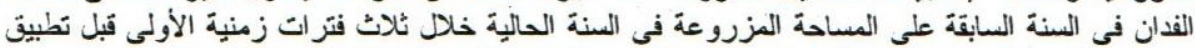

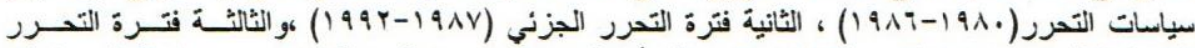

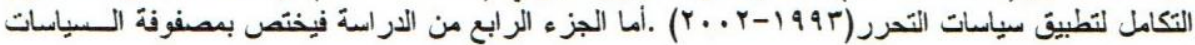

\section{أولا : بيان الثر السياست الززراعية على مساحة وإنتاجية وإنتاج المحاصيل المدروسة.}

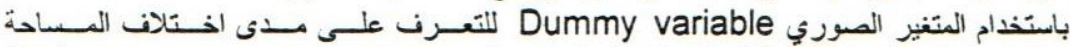

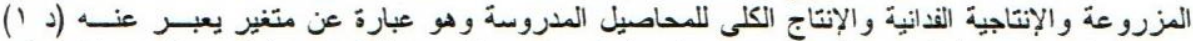

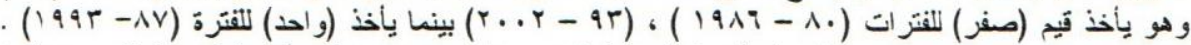

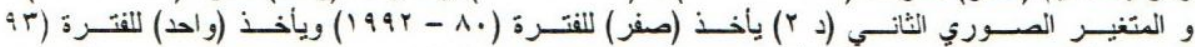

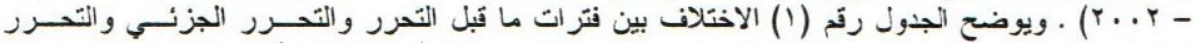

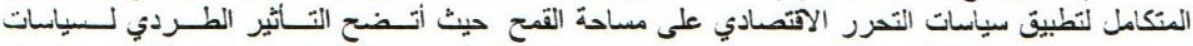

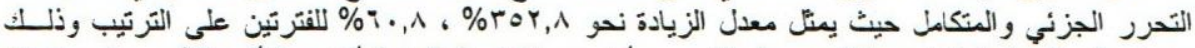

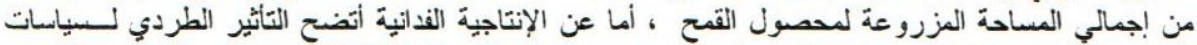

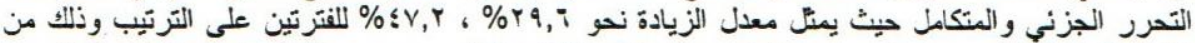

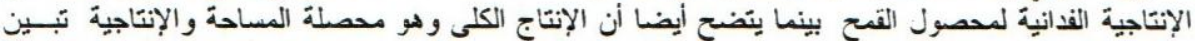

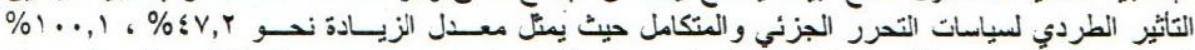

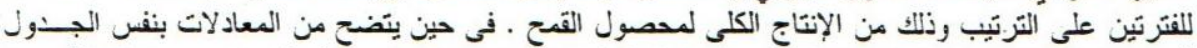

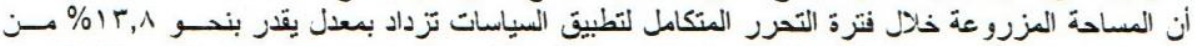

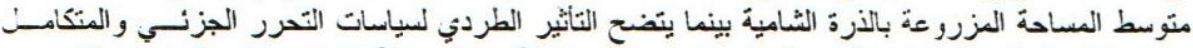

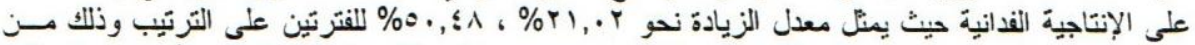

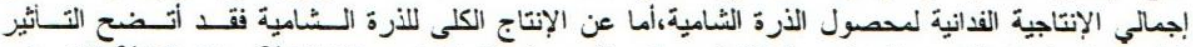

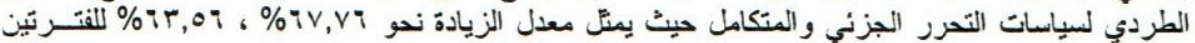

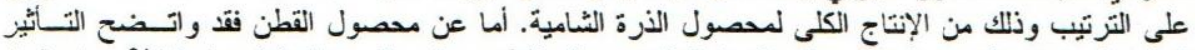

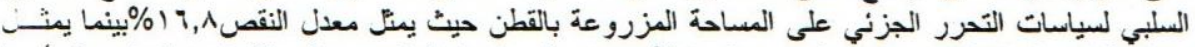

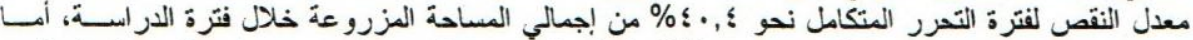

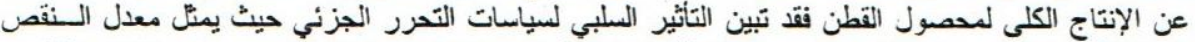

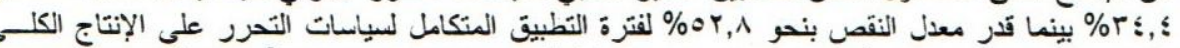

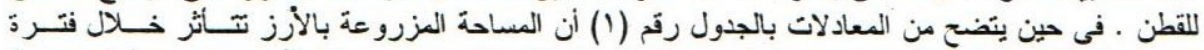

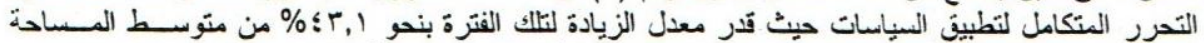

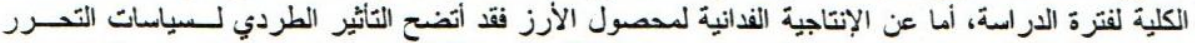

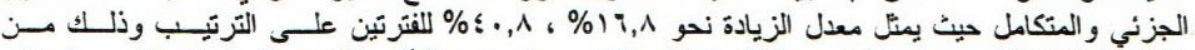
الإنتاجية الفدانية لمصصول الأرز ، وكذلك الإنتاج الكلى حيث تبين التأثير الطردي لسياسات التحرر الجزئي 
Aly, A. I.

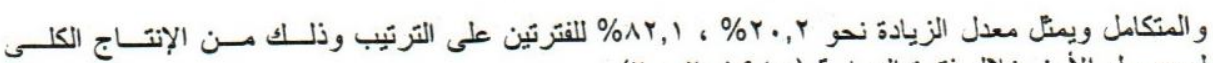

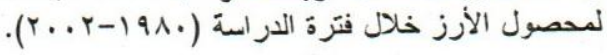

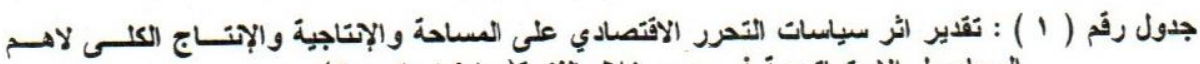

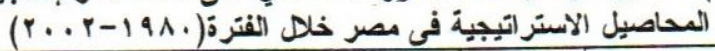

\begin{tabular}{|c|c|c|c|c|c|c|c|c|}
\hline الإحصائية & ف & נر & ت & ت1 & rd & 12 & 1 & البيان \\
\hline$\bullet$ & $01,0 \leqslant$ & $\cdot, \mathrm{AT}$ & $1 \cdot, 1$ & $\varepsilon, \xi T$ & 1100,9 & erA,V & $1 Y \Lambda \leq, r$ & مساحة القمح \\
\hline$\cdots$ & $V V, 01$ & $\cdot, 19$ & $1 r, \xi$ & $I V, r \leq$ & $7, \leqslant \mathrm{~V}$ & $\{, \cdot 7$ & $9,9 \leq$ & الإِتاجيةُ القَمح \\
\hline$\cdots$ & 99,1 & $\cdot, \wedge \mathrm{V}$ & $11, \mathrm{~V}$ & $0, r$ & $r v \leqslant r \wedge, r$ & IrarA, & IrVYV,q & الإنتاج الكلى للقَح \\
\hline$\div$ & 11,1 & $\cdot$, Or & $\xi, \bullet V$ & $1, \leqslant 1$ & $r V V, \varepsilon$ & $90,0 \mathrm{~V}$ & 1179,1 & مساحة الأرة الششامية \\
\hline$\cdots$ & $\leqslant r, i v$ & $\cdot, \mathrm{V9}$ & $9, Y Y$ & $r, T r$ & $\wedge, \wedge \uparrow$ & $r, 79$ & 19,90 & الإتتاجية الذرةٌ المشاميةً \\
\hline$\cdots$ & 07,70 & $\cdot, \wedge \varepsilon$ & $1 \cdot, \leqslant T$ & $r, V !$ & YrTTo, & ATYY,O & $r \leqslant 17 \leqslant, 1$ & إجلتَتاج الكلى للأزرة الشامبة \\
\hline$\cdots$ & $r \leqslant, \Lambda$ & $\cdot, \times 1$ & 7,97 & $r, V T$ & rio, & $10 r, 1 \leqslant-$ & $1 \cdot 9 \cdot, r$ & مساحةً القطن \\
\hline$\bullet$ & $r, 97$ & $\cdot, r 1$ & $1, \wedge \mathrm{V}$ & $r, 10$ & $\cdot, \wedge 9-$ & $1, .9-$ & $\Lambda, r$ & الإتتاجية للفططن \\
\hline$\ddot{*}$ & $r \leqslant, V T$ & $\cdot, \mathrm{VI}$ & 7,99 & $\leq, r$ & $r i .0,0-$ & rTO.,r- & 19!9,0 & الإنتاج الكلى للقططن \\
\hline$\cdots$ & $7 V, 9$ & $\cdot, \mathrm{AV}$ & $1 \cdot, 1$ & 1,0 & Orr,o & $\vee 9,10$ & $9 \wedge r, 1$ & مساحة الأرز \\
\hline$\cdots$ & 09,9 & $\cdot, \wedge T$ & $1 \cdot, 1$ & $\xi, 11$ & $1, r \leq$ &., 01 & $r, r q$ & الإنتاجية الأرز \\
\hline$\cdots$ & $v \cdot, v$ & $\cdot$, AV & $11, r$ & $r, T$ & TITT,q & $V \gamma \cdot, \Lambda$ & TTOV,O & الإحتاج الكلى الأرز \\
\hline
\end{tabular}

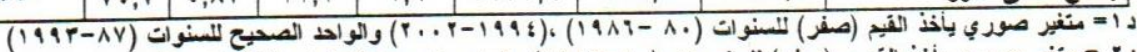

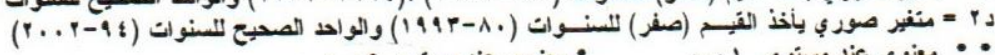

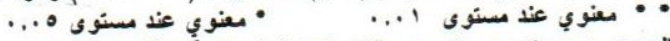
المصدر : جست وحسبت من بياتات الإدارة المركزيةً للاقتصاد الززراعي.

ثانيا : علاقة الأسعار المزرعية بتكلفة الموحدة المنتجة للمحاصيل المدروسة.

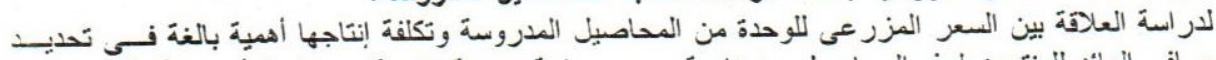

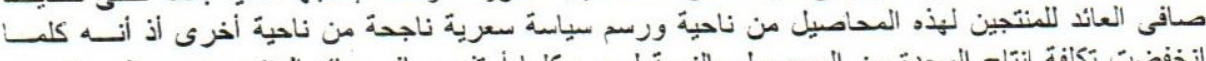

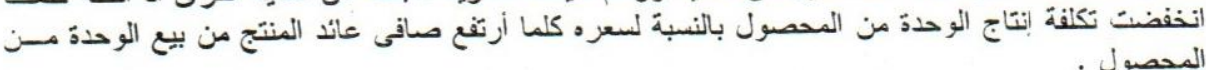

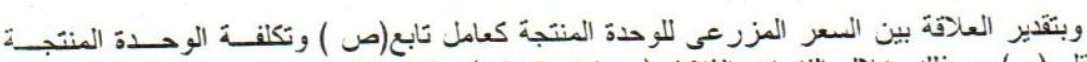

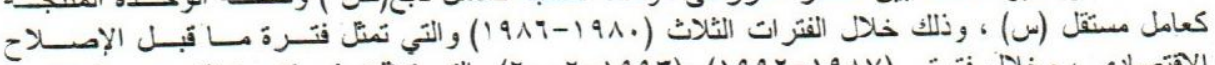

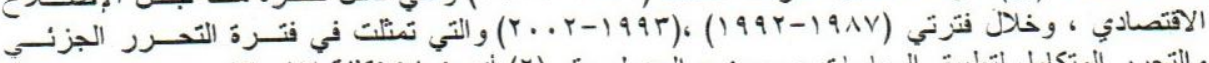

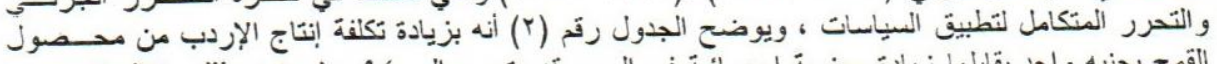

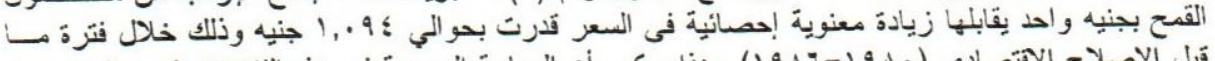

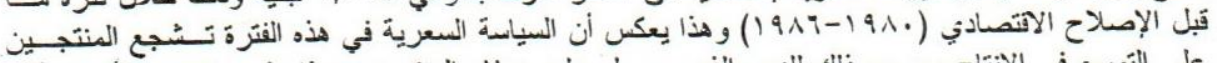

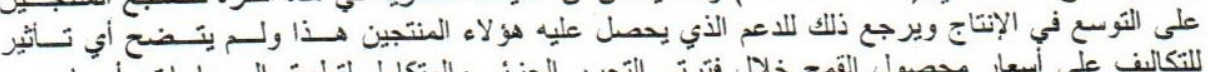

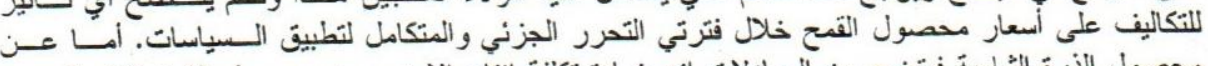

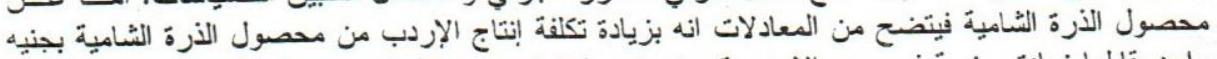

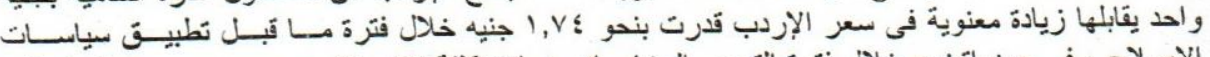

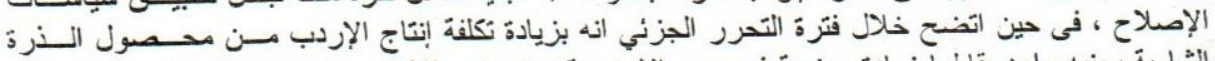

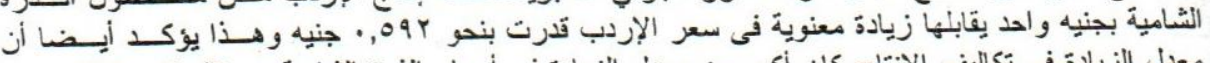

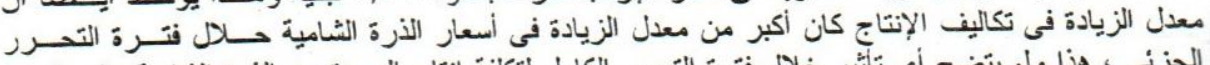

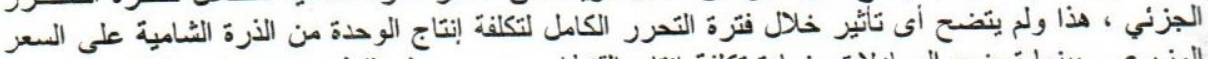

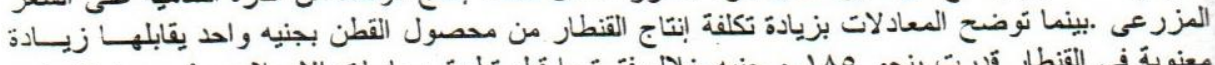

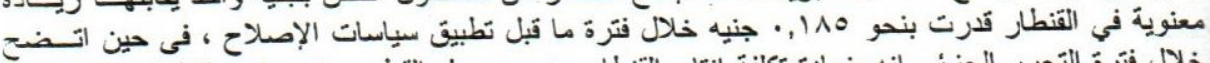

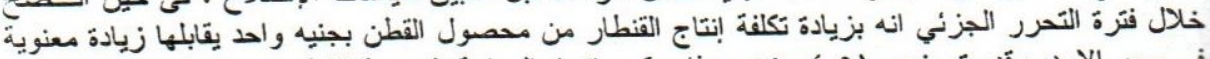

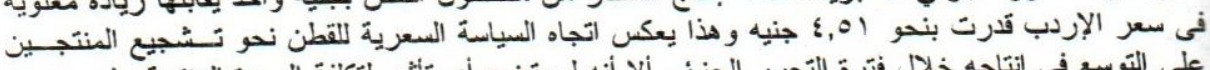

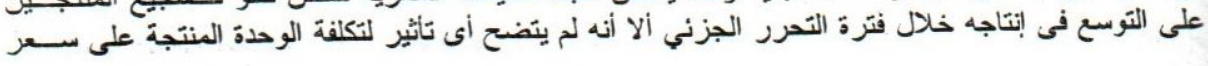




\section{J. Agric. Sci. Mansoura Univ., 30 (7), July, 2005}

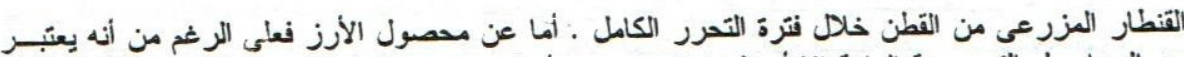

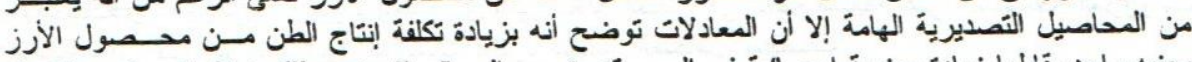

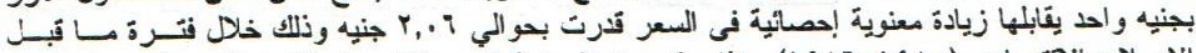

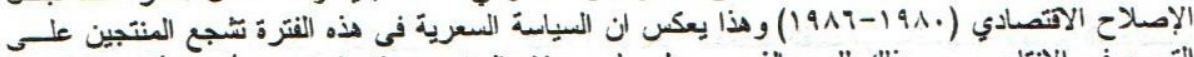

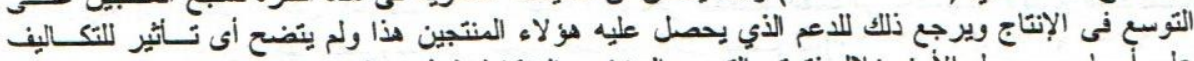

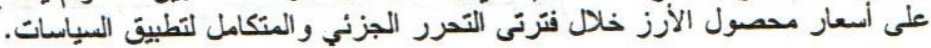

جدول رقم (ץ): العلاقة بين الأسعار المز رعية للوحدة بتكليف إنتاجها لأهـــم المحاصسيل الاســتراتيجية

\begin{tabular}{|c|c|c|c|c|}
\hline الإحصانية (ن) & التحليد & قَتيمة & المعادلة & 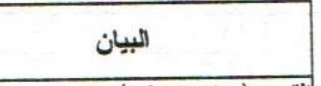 \\
\hline$\because \leqslant r, 0 \leqslant$ & $\cdot, 19$ & 7,09 & صـ, & 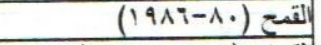 \\
\hline$\leq, 1$ & $\cdot, \leqslant \leqslant$ & $r, \cdot r$ & صر & التقد (199Y-19AV) \\
\hline$\$, 79$ &., 01 & $1, i r$ & صد, = & (r..r-199r) \\
\hline$\cdot 71$ &., 95 & $\Lambda, Y \xi$ & 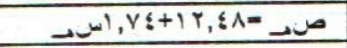 & 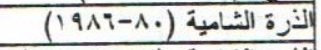 \\
\hline$\bullet 0, r \leq$ & $\cdot, 0 \mathrm{~V}$ & $r, r q$ & صد = = & اللذزة الثامية (9AY-19v) \\
\hline.,$i 0$ & $\cdot, \cdot 9$ & $\cdot, \lambda$ & ص & 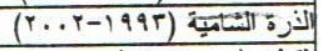 \\
\hline - TVV,AT & $\cdot, 10$ & $0, r V$ & صن, = & 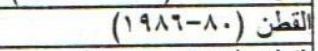 \\
\hline$\bullet 1 \leq, r$ & $\cdot, \mathrm{VA}$ & $r, v \wedge$ & صن_ & 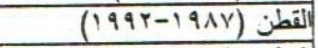 \\
\hline$\cdot, T T$ &., .0 & $\cdot$, OV & صند =r, & القطن (r..r-199r) \\
\hline $09, .0$ & $\cdot, i \leq$ & $r, \cdot v$ & صג & $(19 \lambda i-\lambda \cdot) j j \mid$ \\
\hline$\cdot, V T$ &., 10 & $\cdot, \lambda 0$ & صد, & $(1994-19 \lambda v) j$ \\
\hline$r, 1$ & $\cdot, r 1$ & $1, £$ & صر = & $(r \ldots r-199 r)$ j,y \\
\hline
\end{tabular}

المصدر : حسبت من بيتات ، وزارة الززراعة ، الإلارد المركزية للانتصاد الزراعى .

ثالثا : علاقة المساحة المزروعة الحالية بالعائد الفداني للسنة السابقة للمحاصيل المدروسة.

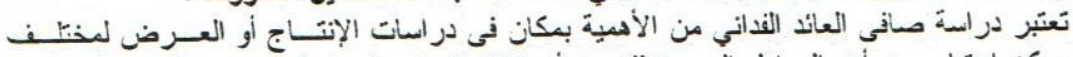

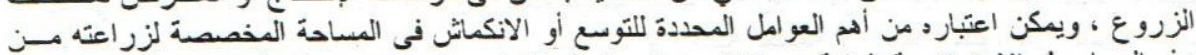

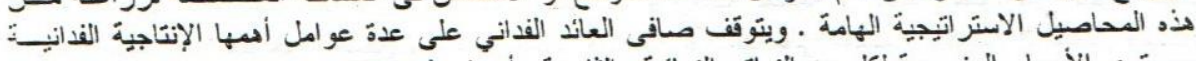

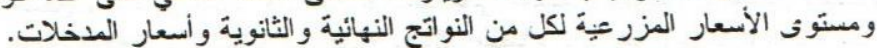

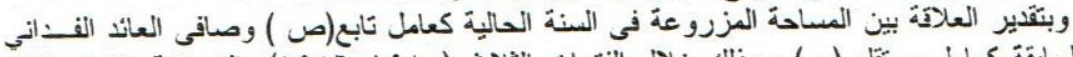

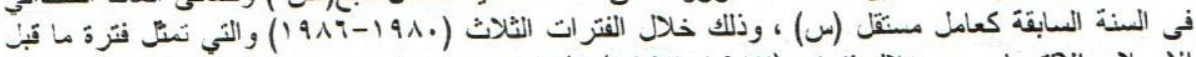

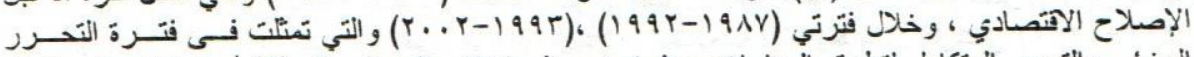

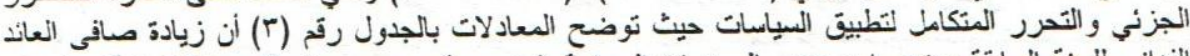

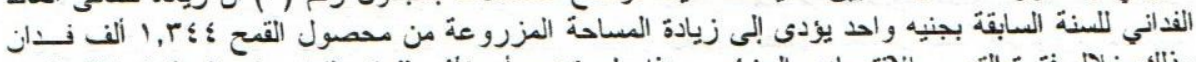

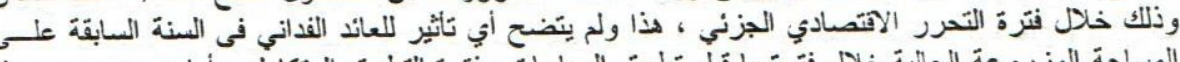

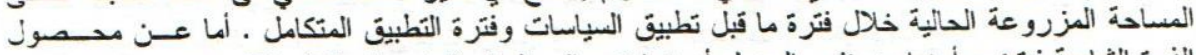

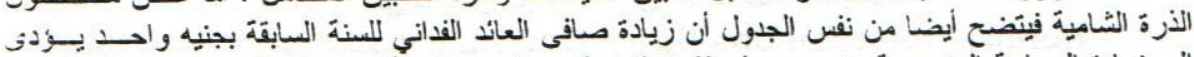

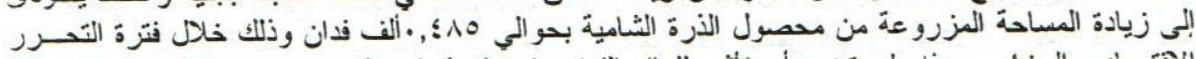

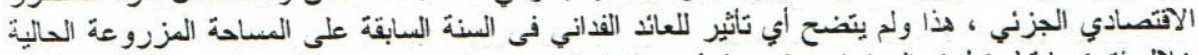

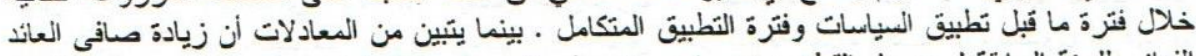

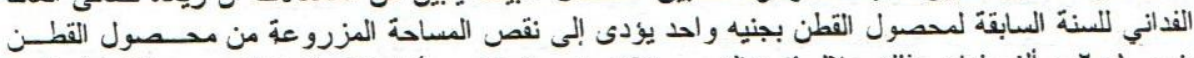

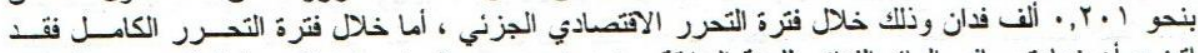

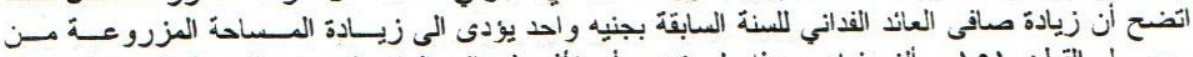

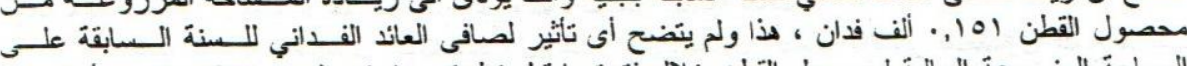

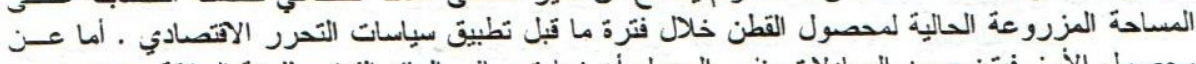

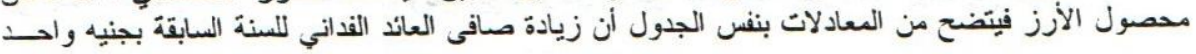




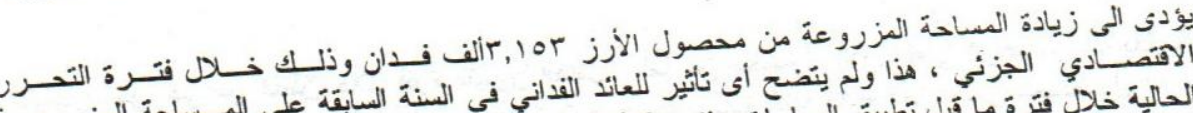

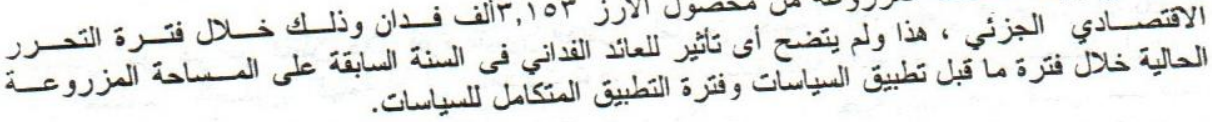

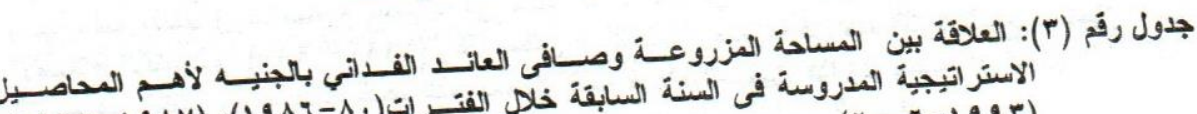

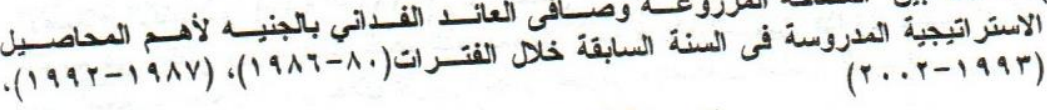

\begin{tabular}{|c|c|c|c|c|}
\hline الإحصنوية (نسية & التحليد & قَيقة & المعادلة & البيان \\
\hline$\leq, \leq \mathrm{V}$ & $\cdot, \leqslant \mathrm{V}$ & $r, 1 Y$ & صد = & $(19 \lambda \uparrow-\lambda \cdot)=0$ \\
\hline$\bullet 104, \mathrm{r}$ & $\cdot, 9 \mathrm{~V}$ & $1, T, T$ & صن & $(199 r-19 \lambda v)=0$ \\
\hline$r, i 0$ &., 10 & $1, \pi r$ & صر & $(r \cdot r-199 r)=0$ (r) \\
\hline $1, \mathrm{NV}$ & $\cdot, \mathrm{rV}$ & $1, T r$ & صر & 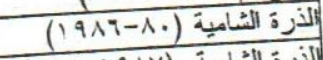 \\
\hline$\cdot 11, . \varepsilon$ &., $\mathrm{VT}$ & $r, T r$ & صـ & (9qr-19Av) \\
\hline.,$i \leq$ & $\cdot, \cdot V$ & $\cdot, \Lambda$ & ص & 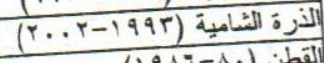 \\
\hline 1,5 &.,$Y T$ & $T, r$ & صر & $(19 \lambda i-\lambda \cdot)$ (1) \\
\hline$\cdot 1 v, \varepsilon$ &.,$\wedge 1$ & $\{, 1 V$ & ص ص & (القطز (199r-191r) \\
\hline$\cdot 11, r$ & $\cdot, 79$ & $\left\{, T^{7}\right.$ & a & القططز (r..r-199r) \\
\hline$\because, Y$ & $\therefore, \ldots 0$ &., $1 \mathrm{~V}$ & صu, & الأزئ) \\
\hline$\because A \theta_{1} z$ & 0,90 & $9, r \leq$ & ص ص & $(199 r-19 \wedge v) j j$ \\
\hline$r_{1}$, & $\cdot, Y 1$ & 1,5 & صـ & (r..r-199r) الأزي \\
\hline
\end{tabular}

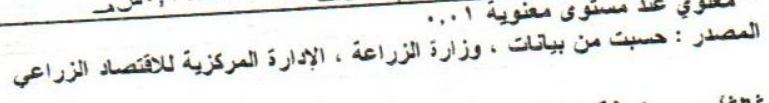

تُاثكا: : مصفوفة تحليل السباسات الززراعية :

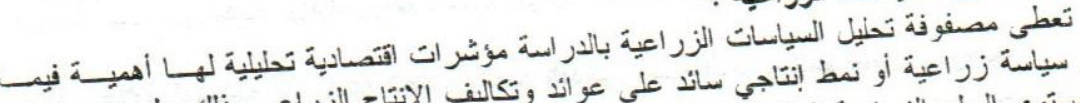

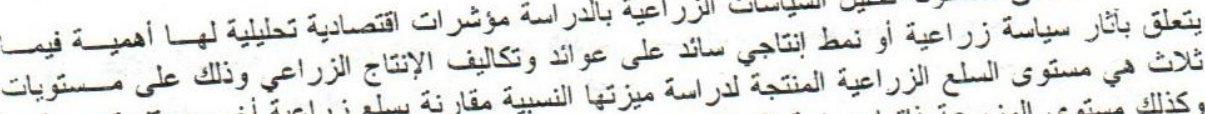

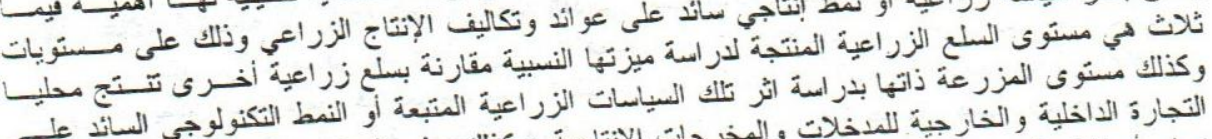

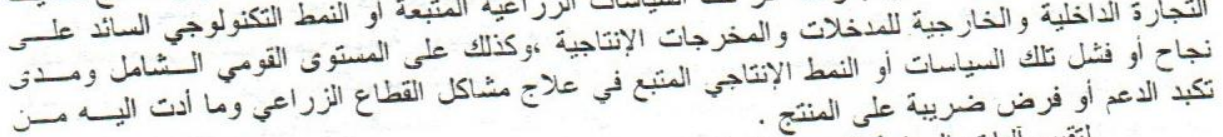

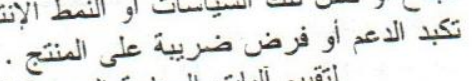

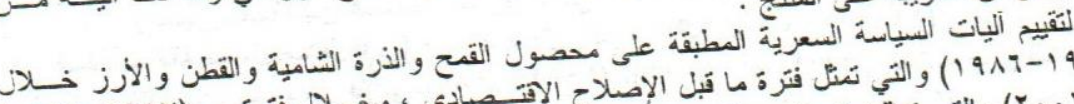

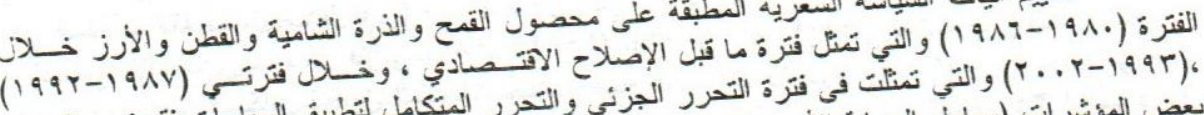

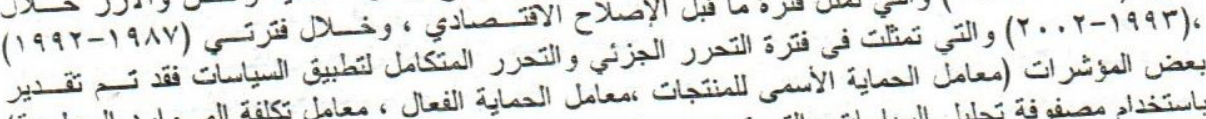

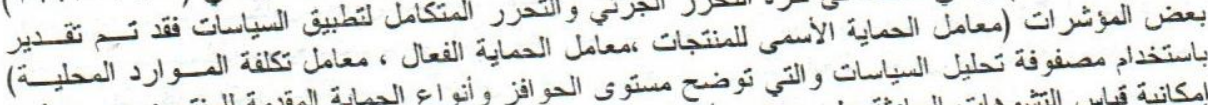

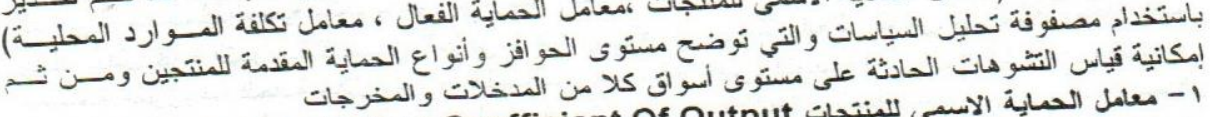

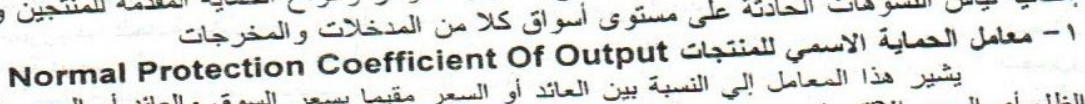

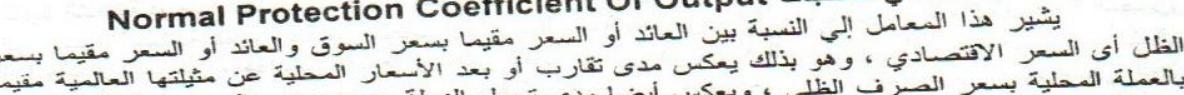

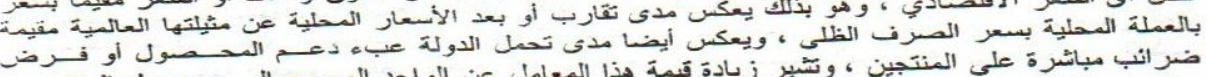

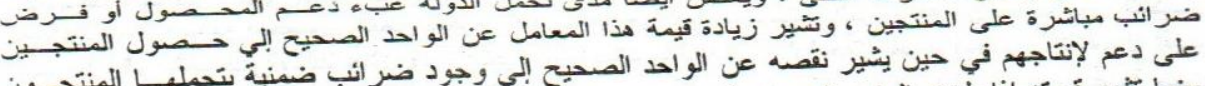

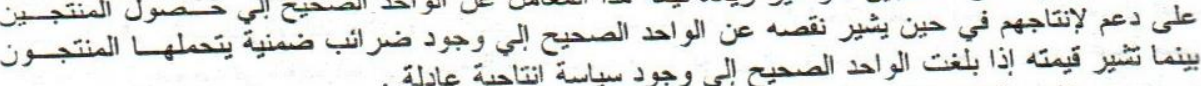

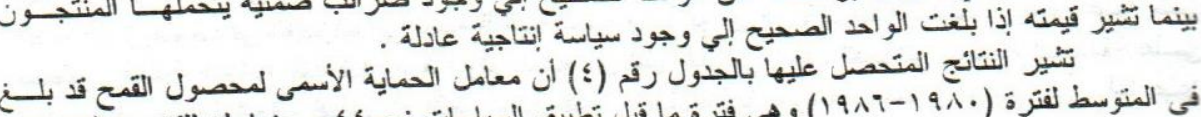

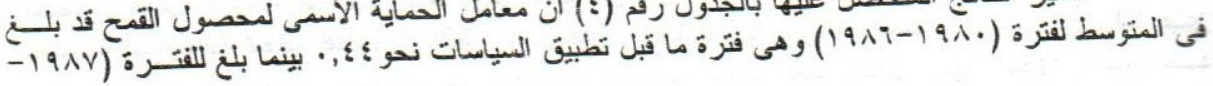




\section{J. Agric. Sci. Mansoura Univ., 30 (7), July, 2005}

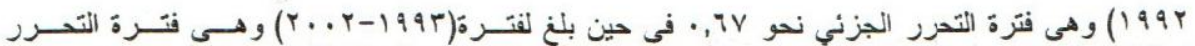

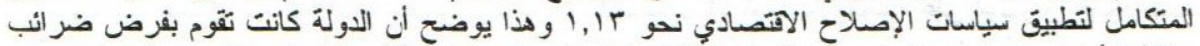

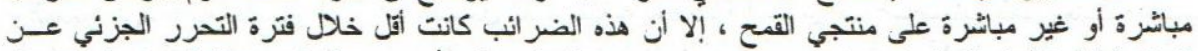

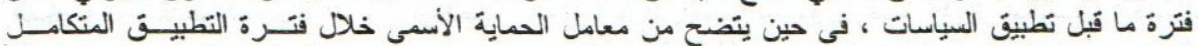

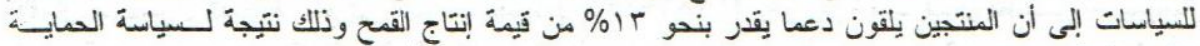

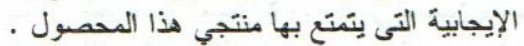

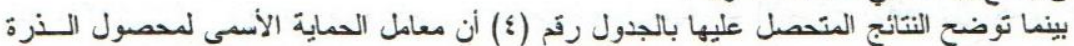

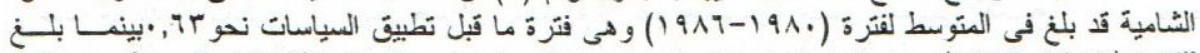

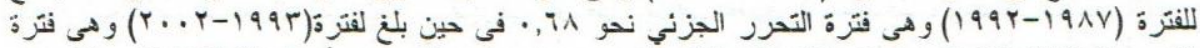

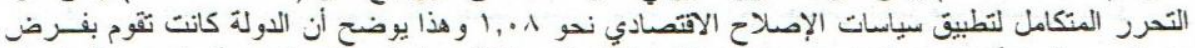

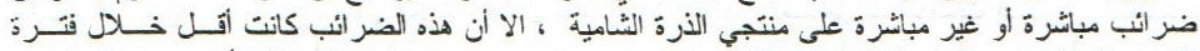

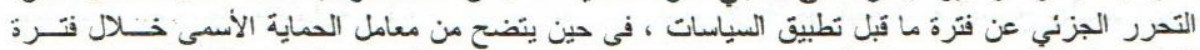

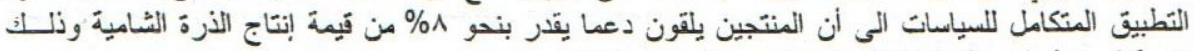

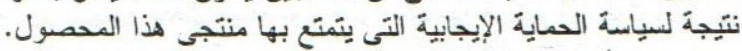

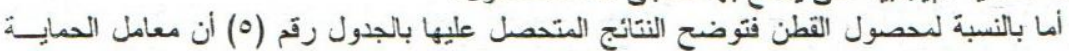

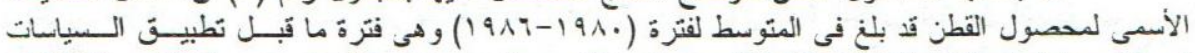

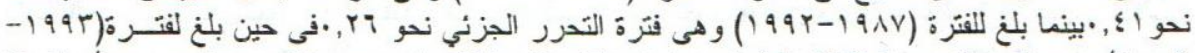

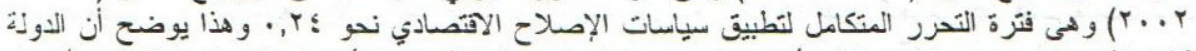

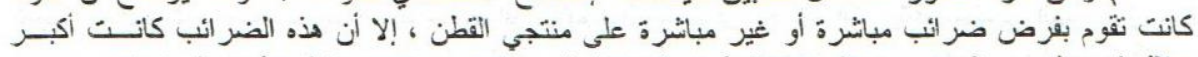

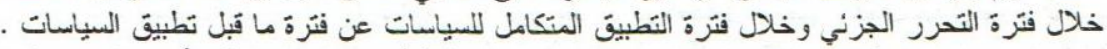

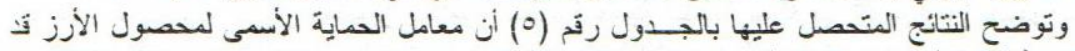

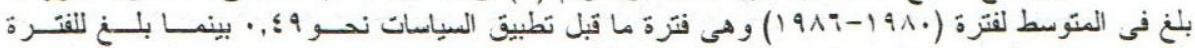

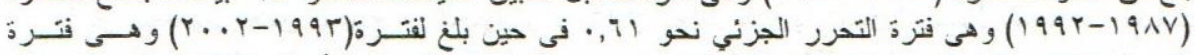

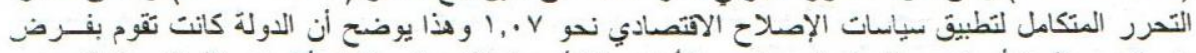

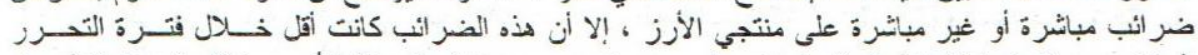

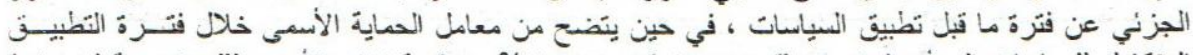

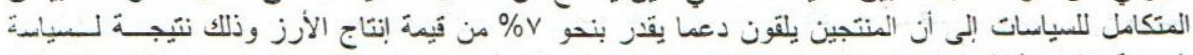

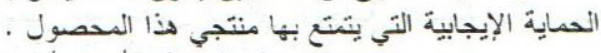

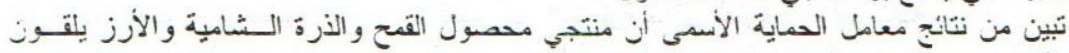

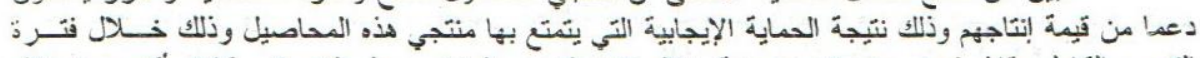

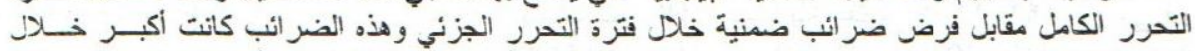

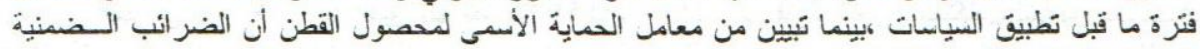

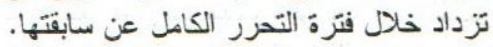

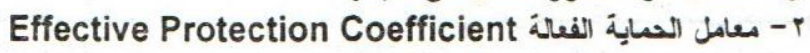

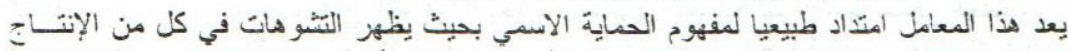

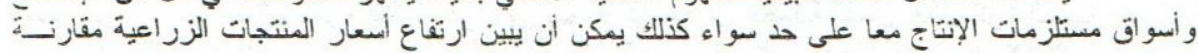

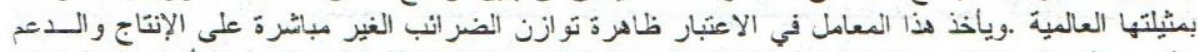

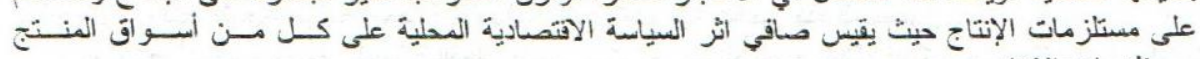
وبصفة عامة فأن معامل الحماية الفعال هو النسبة بين القيمة المضافة عند الأسعار المحلية السائدة

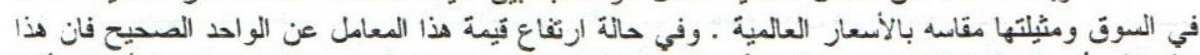

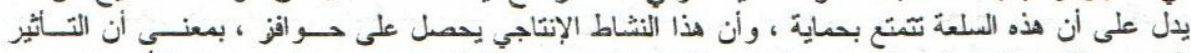

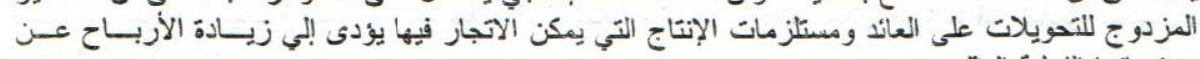

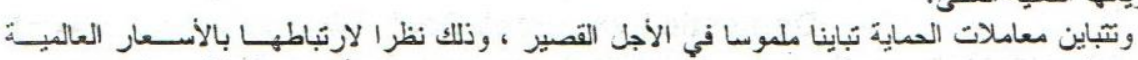

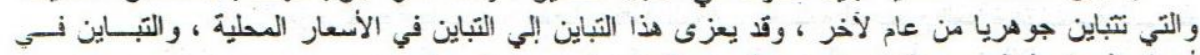

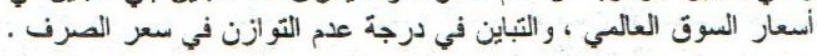




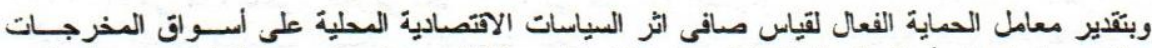

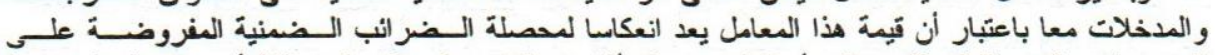

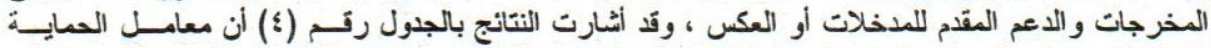

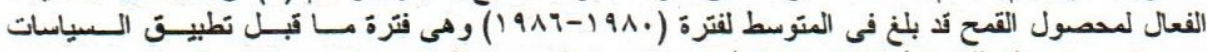

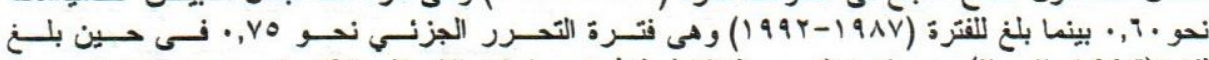

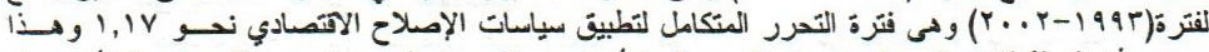

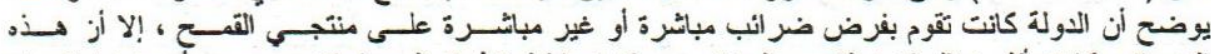

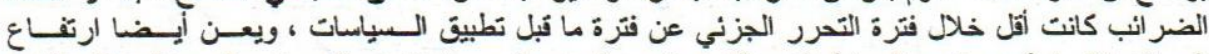

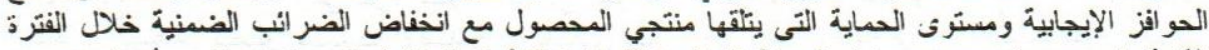

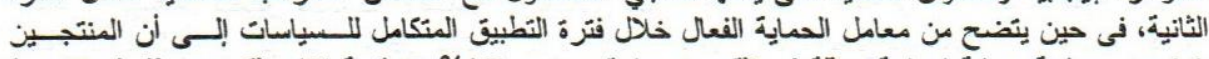

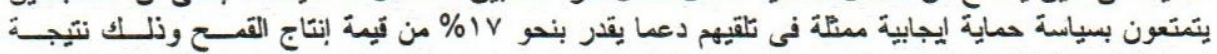

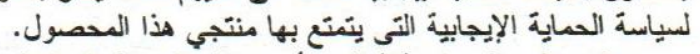

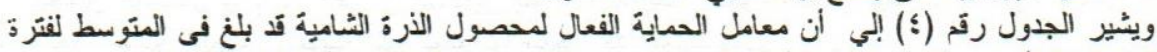

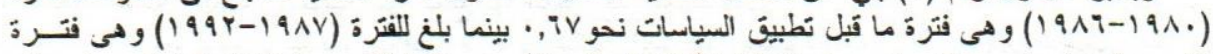

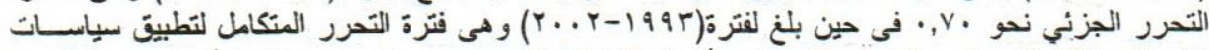

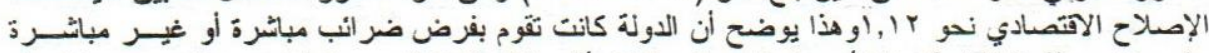

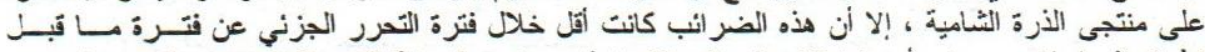

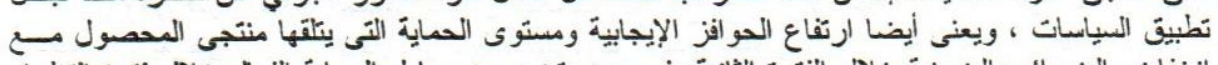

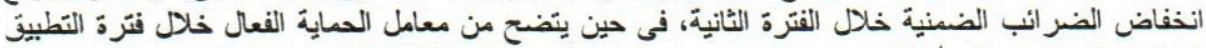

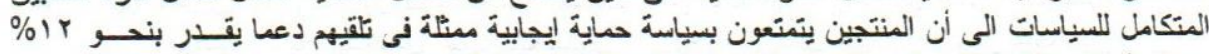

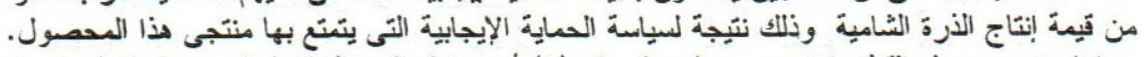

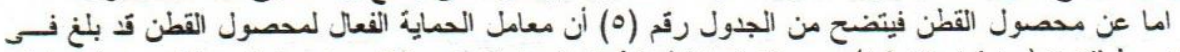

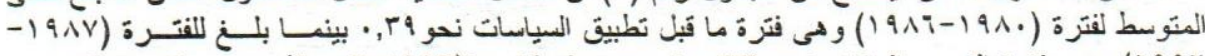

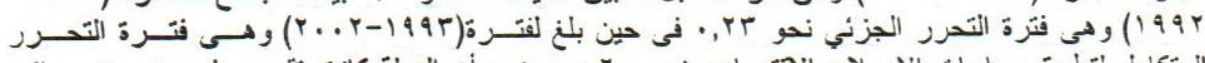

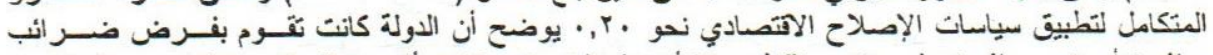

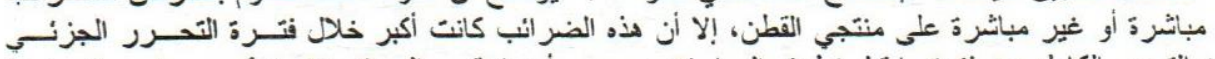

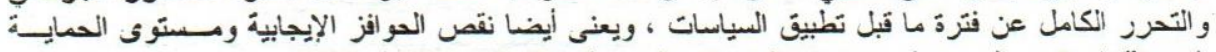

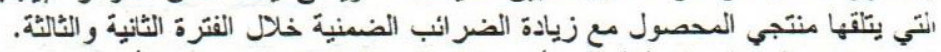

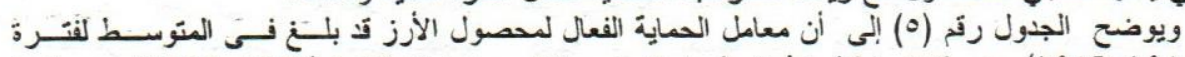

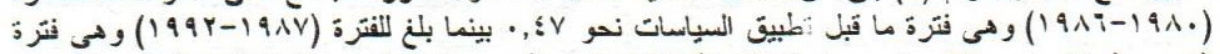

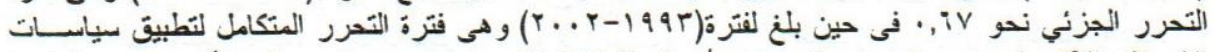

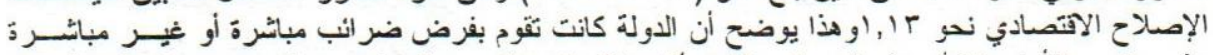

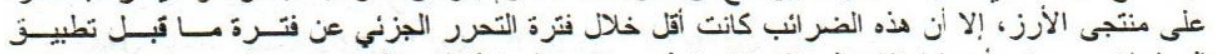

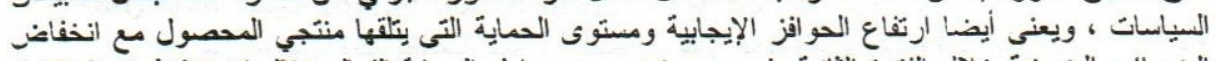

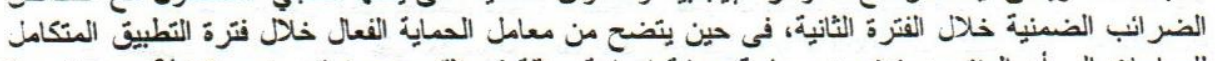

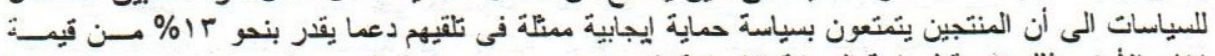

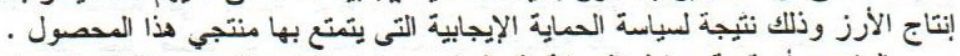

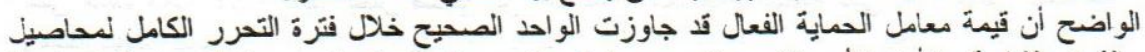

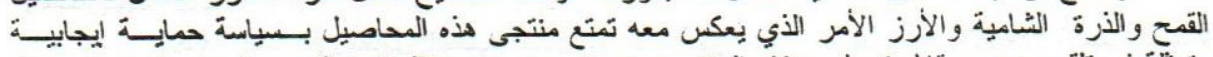

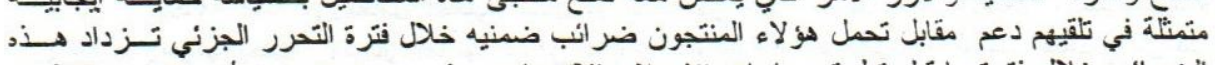

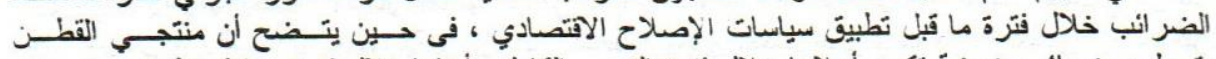

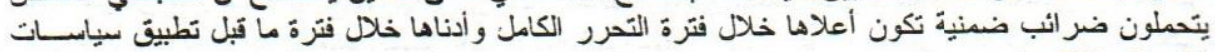

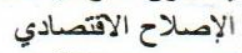

r- معامل تكلفة الموارد المحلية مemestic Resources Cost

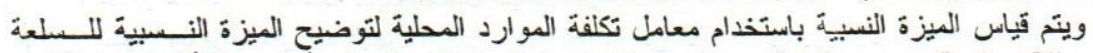

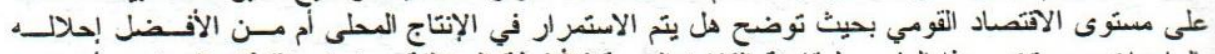

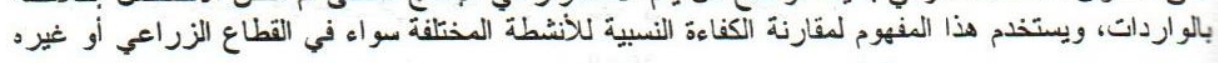




\section{J. Agric. Sci. Mansoura Univ., 30 (7), July, 2005}

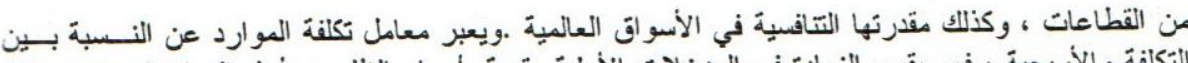

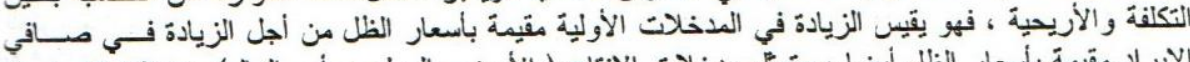

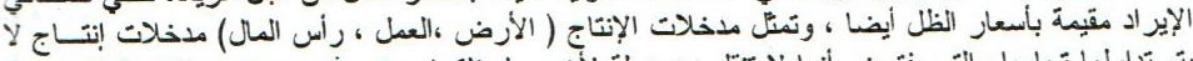

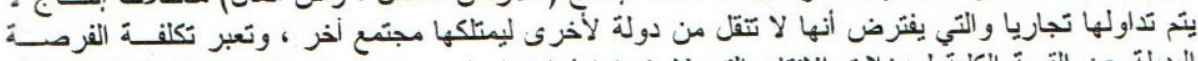

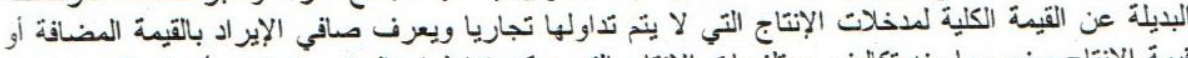

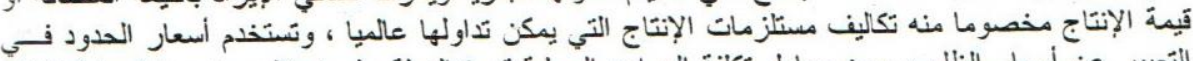

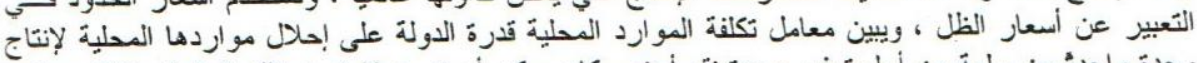

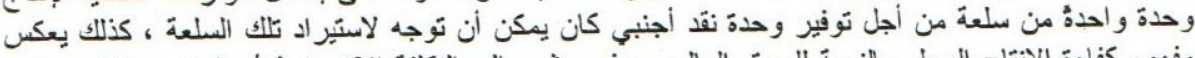

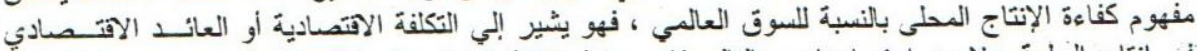

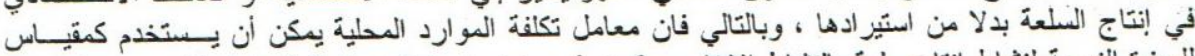

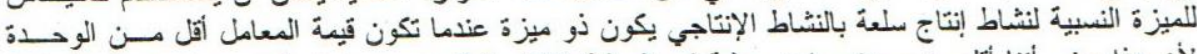

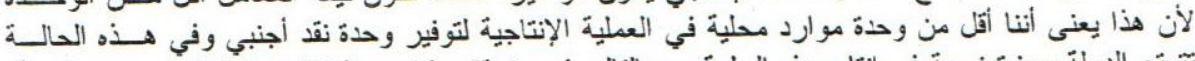

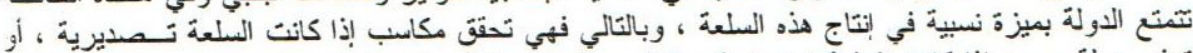

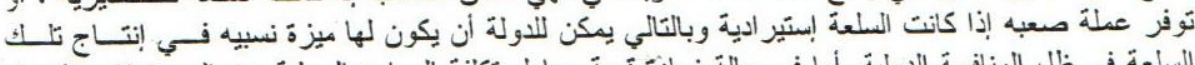

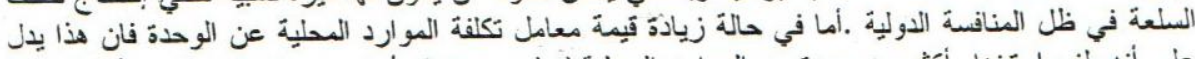

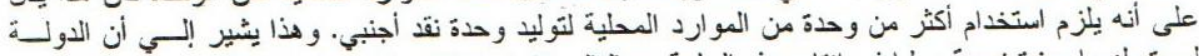

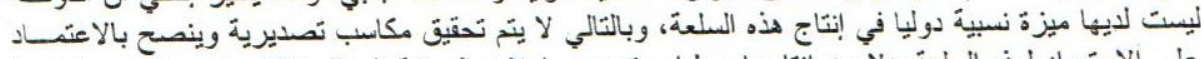

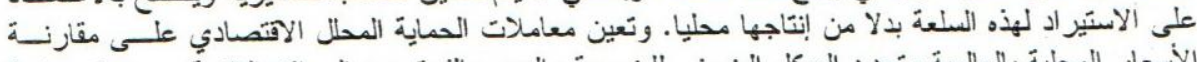

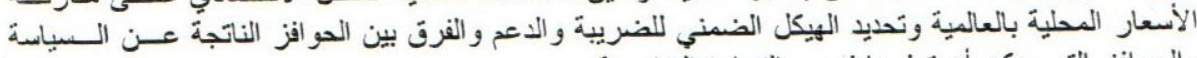

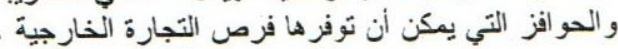

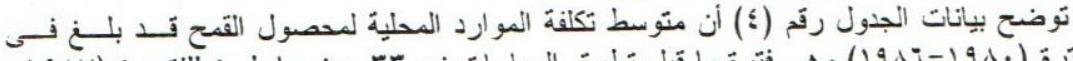

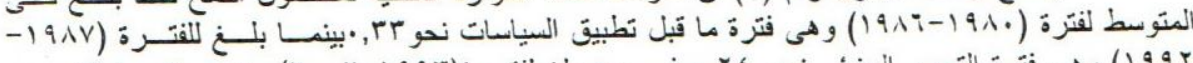

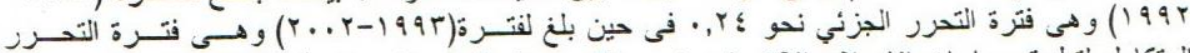

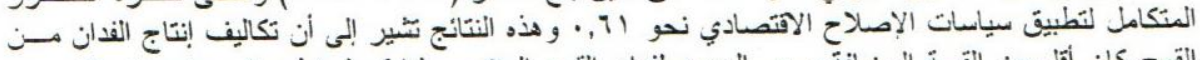

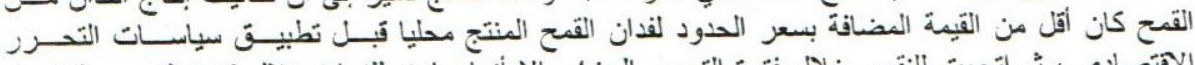

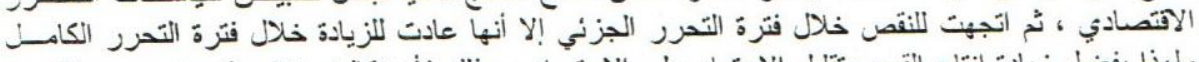

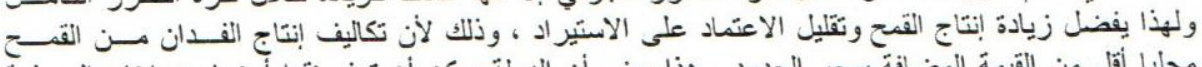

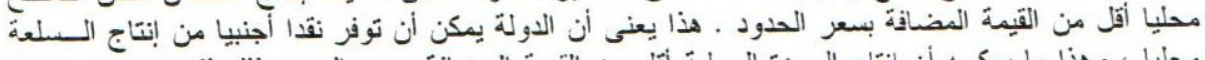

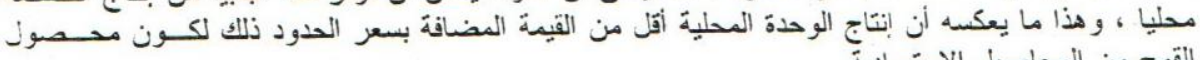

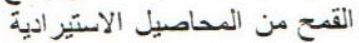

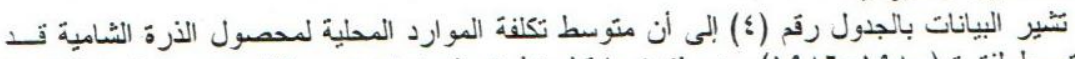

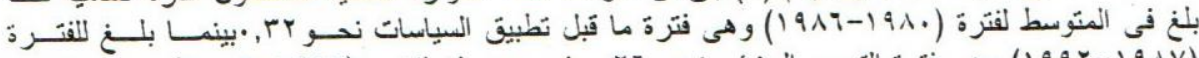

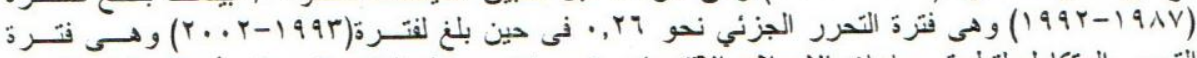

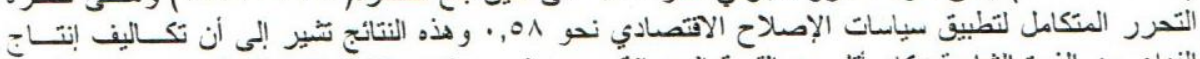

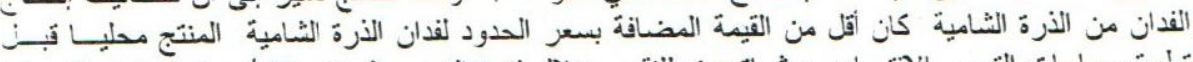

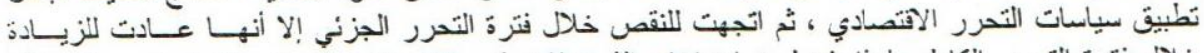

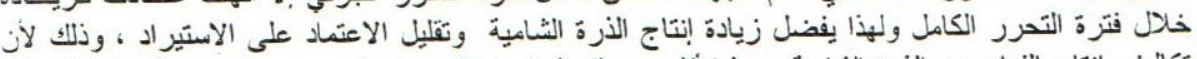

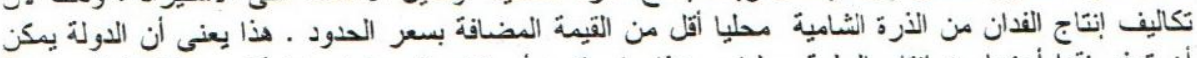

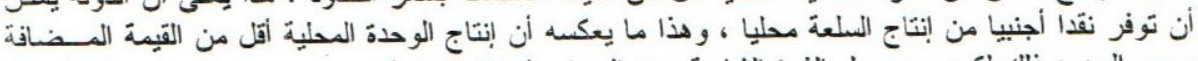

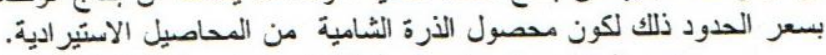

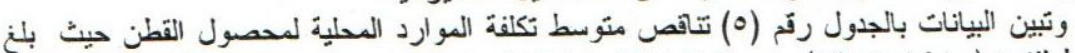

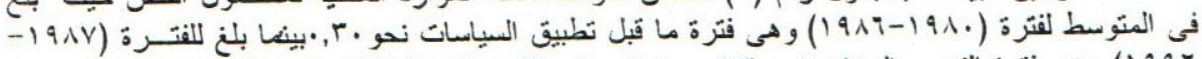

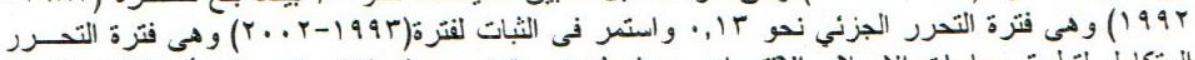

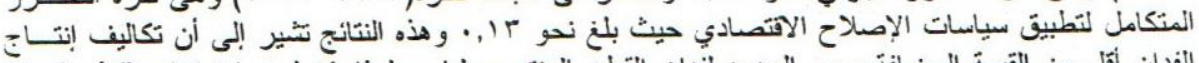

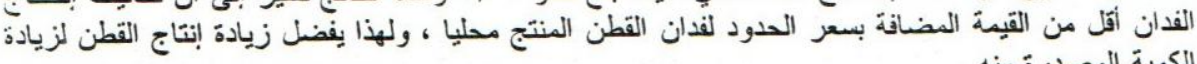

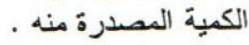


Aly, A. I.

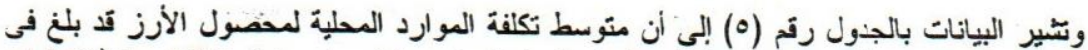

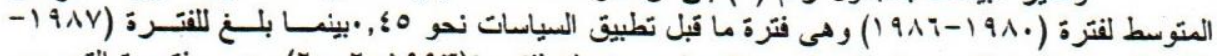

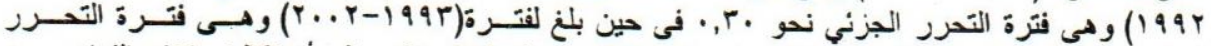

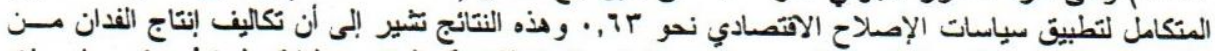

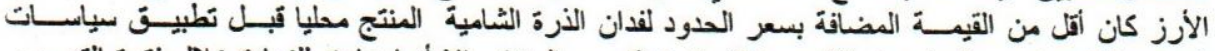

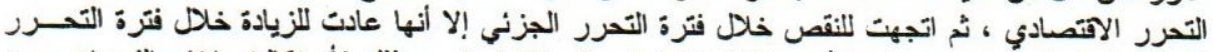

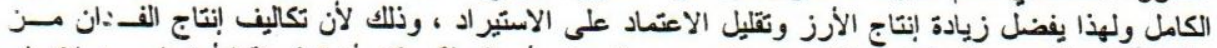

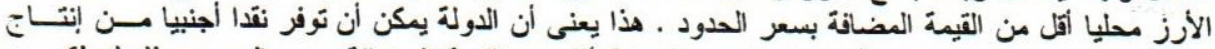

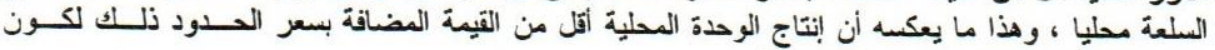
محصول الأرز من المحاصبل ولئ التصديرية.

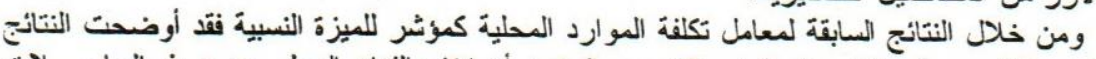

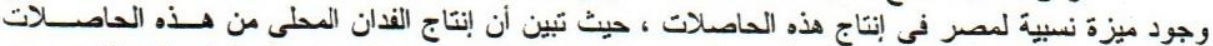

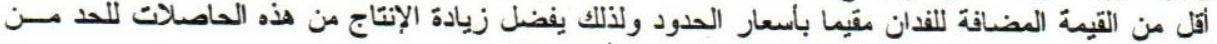

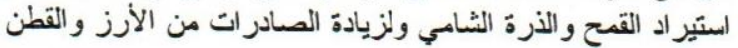

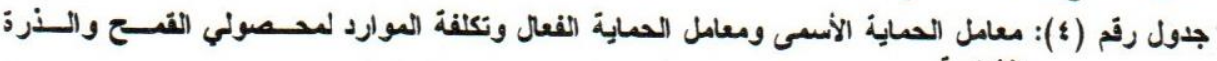
الشثامية

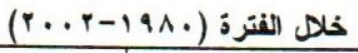

\begin{tabular}{|c|c|c|c|c|c|c|}
\hline \multicolumn{3}{|c|}{ الأرة الشامبة } & \multicolumn{3}{|c|}{ القَح } & \multirow[b]{2}{*}{ السنوات } \\
\hline معامل تكلفة & معامل الحعاية & معامل الحماية & معامل تكلفة & معامل الحماية & معامل الحماية & \\
\hline$\cdot, \Gamma 1$ & $\cdot, \lambda$ & $\cdot, \lambda Y$ & $\cdot, \mathrm{ro}$ &., 01 & $\cdot, 0$ & 191. \\
\hline$\cdot, r \leq$ & $\cdot, r$ & $\cdot, \mathrm{rV}$ & $\cdot, r 0$ &., 01 & $\cdot, r v$ & $19 \lambda 1$ \\
\hline$\cdot, r \leq$ & $\cdot, \leqq Y$ & $\cdot, \leqslant \mathrm{V}$ & $\cdot, r o$ & $\cdot, \leqslant T$ & $\cdot, r$ & 1914 \\
\hline.,$r i$ & 1 & $\cdot, 9$ & $\cdot, \mathrm{rq}$ & $\cdot, i 1$ & $\cdot, \leqslant 7$ & $19 \lambda T$ \\
\hline., 21 & $\cdot, T r$ & $\cdot, i$ & $\cdot, \underline{5}$ & $\cdot, i r$ & $\cdot, \leqslant 0$ & $19 \lambda !$ \\
\hline$\cdot, \mathrm{rV}$ & $\cdot, \lambda r$ & $0,7 i$ & $\cdot, \mathrm{TY}$ & $\cdot, 10$ & $\cdot, \ldots \leqslant$ & 1910 \\
\hline$\cdot, r$ & $\cdot, \mathrm{VT}$ & $\cdot, 01$ &.,$T 0$ & $\cdot, \lambda$ & $\cdot, 00$ & 1918 \\
\hline$\cdot, \Gamma !$ & $\cdot, \mathrm{iv}$ & $\cdot, \mathrm{ir}$ &.,$r r$ & $\cdot, i$ & $\cdot, \leq \leqslant$ & المتوسط \\
\hline$\cdot, r Y$ & $\cdot, i !$ & $\cdot, 0 Y$ & $\cdot, r V$ & $\cdot, \uparrow \wedge$ & $\cdot, \leqslant i$ & $19 \lambda \mathrm{V}$ \\
\hline$\cdot, r$ & $\cdot, \leqslant\}$ & $\cdot,+79$ & $\cdot, 11$ & $\cdot, \leqslant 0$ & $\cdot, r_{1}$ & $19 \lambda 1$ \\
\hline., $1 i$ & $\cdot, \theta r$ &., 01 &., $1 T$ & $\cdot, \div 9$ & $\cdot, \leqslant 0$ & 1919 \\
\hline$\cdot, r$ & $\therefore, v e$ & $\cdot, \mathrm{Ve}$ & $\cdot, r$ & $\cdot, \mathrm{V} \leq$ & $\cdot, 79$ & 199. \\
\hline$\cdot, r !$ & $\cdot, 9 !$ & $\cdot, 90$ &.,$r !$ & $1, \cdot v$ & $1, \cdot 1$ & 1991 \\
\hline $0, r i$ &., 90 & $\cdot ., 9 v$ & $\cdot, T r$ & $1, \cdot \leq$ & $1, . \leq$ & 1997 \\
\hline.,$P i$ & $\cdot, \mathrm{V}$ &., $\mathrm{in}$ & $\cdot, r \leq$ &., $\mathrm{V}=$ & $\cdot, i \mathrm{Y}$ & المتوسط \\
\hline$\cdot, \leqslant 7$ & $\cdot, \lambda T$ & $\cdot, \lambda \vee$ & $\cdot$, or & $1, \cdot r$ & $1, \cdot r$ & 1994 \\
\hline$\cdot, \leqslant Y$ & $\cdot, 9 \mathrm{~V}$ & $\cdot ., 91$ & $\cdot, i \leq$ & $1, r 0$ & $1, r r$ & 1995 \\
\hline$\cdot, \mathrm{ro}$ &., $9 !$ &., 97 & $\cdot, \leqslant r$ & $\cdot, \lambda i$ & $\cdot, 94$ & 1990 \\
\hline$\cdot, r i$ & $\cdot, \mathrm{V}$ & $\cdot, \mathrm{VA}$ & $\cdot, r T$ & $\cdot, \mathrm{VV}$ & $\cdot, \wedge !$ & 1997 \\
\hline$\cdot, \leqslant 1$ & $1, \cdot 1$ & $1, \cdot \leq$ & $\cdot, \leqslant 0$ & $1, \cdot 9$ & $1, \cdot v$ & $199 \mathrm{~V}$ \\
\hline 0,79 & $1, r T$ & $1, Y Y$ & $\cdot, 1 \times 0$ & $1, r 0$ & $1, r 0$ & 1991 \\
\hline$\cdot, \lambda$. & $1, r i$ & $1, r 7$ & $\cdot, \mathrm{VT}$ & $1, \leq$ & $1, r Y$ & 1999 \\
\hline$\cdot, 91$ & $1, r !$ & 1,11 & $\cdot, Y 1$ & $1, r 7$ & $1, r \leq$ & $r \ldots$ \\
\hline$\cdot, M$ & $1, \pi 1$ & $1, r$ & $\cdot, v \leq$ & $1, \Gamma Y$ & $1, r o$ & $r \ldots 1$ \\
\hline$\cdot, \wedge V$ & $1, \leqslant r$ & $1, r$ & $\cdot, 87$ & 1,5 & $1, r \Lambda$ & $r \ldots r$ \\
\hline$\cdot, 01$ & $1,1 \mathrm{r}$ & $1, \cdot 1$ & $\cdot, 11$ & $1,1 \mathrm{~V}$ & $1,1 r$ & المتوسط \\
\hline
\end{tabular}

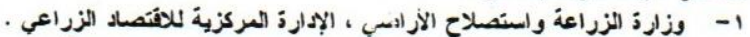

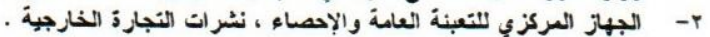


جدول رقم (0) : معامل الحماية الأسمى ومعامل الحماية الفعل وتكلفة الموارد لمحسـصولي القطسـن والأزز

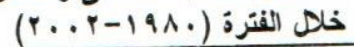

\begin{tabular}{|c|c|c|c|c|c|c|}
\hline \multicolumn{3}{|c|}{ الأرز } & \multicolumn{3}{|c|}{ ن } & \multirow[b]{2}{*}{ السنو ات } \\
\hline معامل تكلفة & معامل الحمايةة & معامل الحمايةً & معامل تكلفة & معامل الحماية' & معامل الحماية & \\
\hline$\cdot, \leqq Y$ & $\cdot, \$ 1$ & $\cdot, 0$ & $\cdot, Y_{0}$ & $\cdot, r q$ & $\cdot, \xi T$ & 191. \\
\hline.,$r 1$ & $\cdot, Y^{q}$ & $\cdot, r i$ & $\cdot, r i$ & $\cdot, \mathfrak{s}$ & $\cdot, \leqslant 1$ & 1911 \\
\hline$\cdot, \Gamma 7$ & $\cdot, r Y$ & $\cdot, \leq$ & $\cdot, \xi$ & $\cdot, \leqslant V$ & $\cdot, 0$ & 1917 \\
\hline., $\mathrm{itr}$ &.,$\leqslant 0$ &., 59 & $\cdot, \leqslant \leq$ &.,$\$ 7$ & $\cdot, \leqslant 9$ & $191 r$ \\
\hline$\cdot, \xi Y$ &., $0 \leqslant$ &., $0 \leq$ & $\cdot, r 1$ & $\cdot, r v$ & $\cdot, \mathrm{rA}$ & 1915 \\
\hline., $0 r$ &., 79 & $\cdot, \bar{T}$ & $\cdot, r T$ &.,$T Y$ & $\cdot, \mathrm{rT}$ & 1910 \\
\hline$\cdot, \$ 7$ &., 71 &., $0 \mathrm{r}$ & $\cdot, r \leq$ &.,$\times 9$ & $\cdot, r 1$ & 1917 \\
\hline$\cdot, \leqslant 0$ & $\cdot, \subseteq \mathrm{V}$ & $\cdot, 59$ & $\cdot, r$ & $\cdot, r q$ & $\cdot, \leqslant 1$ & المنوسط \\
\hline$\cdot, \Gamma Y$ & $\cdot, \leqslant \leq$ & $\cdot, \leq$ & $\cdot, Y 7$ & $\cdot r$ & $\cdot, \Gamma Y$ & $19 \wedge \mathrm{V}$ \\
\hline., 19 &., 00 & $\cdot, \leqslant 7$ & $0,1 \leq$ & $\cdot, 11$ & $\cdot, Y$ & $19 \lambda 1$ \\
\hline., 19 & $\cdot, \leqslant \lambda$ & $\cdot, 89$ & $\cdot, \cdot \wedge$ &., $1 r$ &., 17 & 1919 \\
\hline$\cdot, Y V$ & $\cdot, \pi$ & $\cdot, 7 \leq$ & $\cdot, 1 T$ & $\cdot, Y T$ & $\cdot, r 0$ & 199. \\
\hline$\cdot, \mathrm{rV}$ &., 97 & $\cdot, 91$ &., $1 T$ & $\cdot, \Gamma 7$ & $\cdot, r q$ & 1991 \\
\hline$\cdot, \leqslant T$ & $\cdot, 9 \mathrm{~V}$ & $\cdot, \mathrm{V} \leqslant$ & $\cdot, \cdot 7$ & $\cdot, r$ & $\cdot, Y Y$ & $199 r$ \\
\hline$\cdot, r$ & $\cdot, i v$ & $\cdot, 71$ &., 15 & $\cdot, T r$ & $\cdot, Y T$ & المنوسط \\
\hline., $0 \leq$ & $\cdot, \wedge \mathrm{V}$ & $\cdot, 9$ & $\cdot, \cdot \wedge$ &., 19 & -,$Y T$ & 1995 \\
\hline$\cdot, 01$ & $\cdot, 9 \mathrm{~V}$ & $\cdot .91$ &., 10 & $\cdot, Y Y$ & $\cdot, r V$ & 1995 \\
\hline .,it & $\cdot, 19$ &., 94 &., 10 & $\cdot, r 7$ & $\cdot, \xi$ & 1990 \\
\hline$\cdot, \leqslant 1$ & $\cdot, 99$ &., 99 & $\cdot, \cdot \lambda$ &., 19 & $\cdot, T Y$ & 1997 \\
\hline$\cdot, \leqq \wedge$ & $1, \cdot r$ & $1, T$ &., $1 r$ & $\cdot, r \leq$ & $\cdot, \times 9$ & 1998 \\
\hline$\cdot, 17$ &., 00 & $\cdot$ it &., $1 \leq$ & 0,17 & $\cdot, r$ & $199 \lambda$ \\
\hline$\cdot, \mathrm{V} \mathbf{\xi}$ & 1,70 & 1,6 &., 16 & $\cdot, 17$ & $\cdot, r$ & 1999 \\
\hline$\cdot, i \wedge$ & $1,6 \mathrm{~V}$ & 1,79 & $., 1:$ &., 10 &., 19 & r... \\
\hline., 10 & $1, \leqslant r$ & $1, r$ &., 10 & $\cdot, 11$ & $\cdot, r$ & $r \ldots 1$ \\
\hline$\cdot, \mathrm{V} \leq$ & $1, \leqslant 9$ & $1, \pi 1$ &., 10 &., 10 & $\cdot, 1 \wedge$ & $r \ldots r$ \\
\hline.,$i r$ & $1,1 T$ & $1, \cdot v$ &., $1 T$ & $\cdot, r$ & $\cdot, r \leq$ & المنّوسط \\
\hline
\end{tabular}

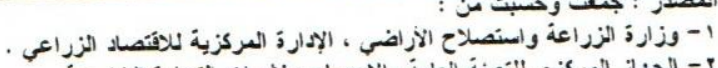

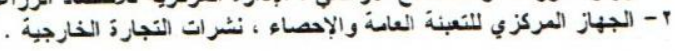

\section{المر اجــ}

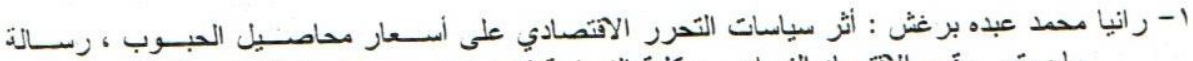

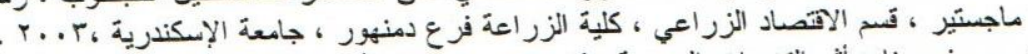

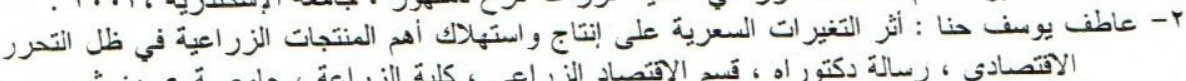

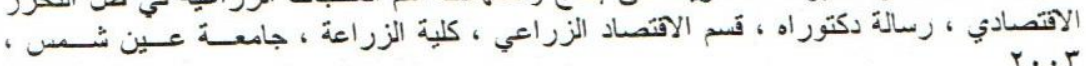

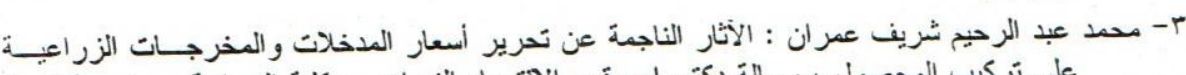

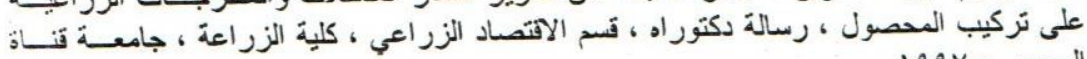
؛- ناجح عبد الجليل أحمد عمر : دراسة تحلبلية لأهم السياسات الافتصادية على استُهلاك بعض محاصـيليل

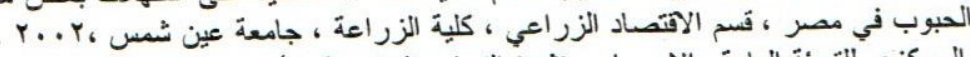

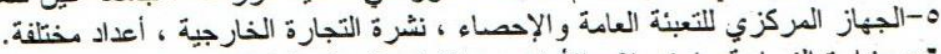

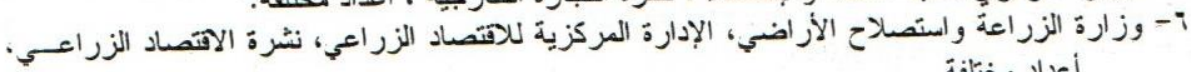
أعداد مختلفة. 
7- Abdel Aziz Ibrahim - The Policy Analysis Matrix for crop Rotations in Egypt Acas study, institute of National , August 1992

\section{THE IMPACT OF AGRICULTURAL POLICIES ON PRODUCING THE MOST IMPORTANT STRATEGICAL CROPS IN EGYPT}

Aly, A. I.

Depe rtment of Agricultural Economics, Faculty of Agric., El - Fayoum

ABSTRACT

The study aims at showing the impacts of the economic liberalization policies on the prices of the most important strategical crops in Egypt which are wheat, corn, rice and cotton, through analyzing the different relations of the liberalization period and the period before liberalization, in order to know the effect of changes in global prices on the local prices of these crops, also to know the impact of canceling the subsidy for the agricultural production requirements. By using the Dummy variable, the direct impact of integrated and partial liberalization policies had been indicated on the production, productivity and the area of wheat, corn and rice crops. While the negative impact had been indicated on cotton

Studying the relation between the farm price for the unit and the productive unit cost, indicated that the price policy encourages the farmers to increase producing wheat, corn and rice during the period before implementing the economic liberalization. It indicated also that the average of increase in cost was more than the average of increase in prices during the period of integrated and partial liberalization for implementing the policies. While it had been indicated that the government encouraged cotton producers only during the period of partial liberalization.

By estimating the relation between the cultivated area and the net profit of the last year, the direct impact of the net profit of the last year had been indicated on wheat, corn and rice areas during the partial liberalization period. The negative impact of cotton during the partial liberalization period had been indicated, while the direct impact of cotton had been indicated during the period of integrated implementation of policies.

The results of nominal protection coefficient indicated that wheat, corn and rice producers receive a subsidy from the value of their $f$-oduction as a result of the positive protection which covers the producers of these crops, during the complete liberalization period in exchange for imposing an indirect taxes during the partial liberalization period. These taxes were greater during the period before implementing the policies. While the nominal protection coefficient for cotton crop indicated that the indirect taxes increase during the period of complete liberalization more than the period before. It is clear that the effective protection coefficient value passed integer one during the period of complete liberalization for wheat, corn and rice crops, this reflects that the producers of these crops are covered with a positive protection policy represented in receiving a subsidy in exchange for imposing an indirect taxes on these producers during the period of partial liberalization, and these taxes increase during the period before implementing the policies. While indirect taxes were imposed on cotton producers, these taxes reach its maximum during the complete liberalization period and reach its minimum during the period before implementing policies. By using the previous results of Domestic Resources cost factor as an indicator, the results indicated that Egypt has a competitive advantage in producing these crops that it was indicated that the production of domestic feddan from these crops is lesser than the added value to the feddan compared with border prices. So it is preferable to increase the production of these crops. 